\title{
Booster Double Harmonic Setup Notes
}

\author{
C.J. Gardner
}

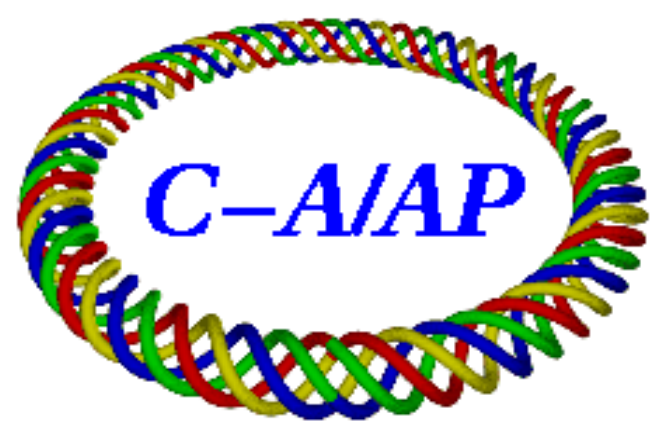

\section{Collider-Accelerator Department Brookhaven National Laboratory Upton, NY 11973}

\section{U.S. Department of Energy \\ Office of Science, Office of Nuclear Physics}

Notice: This document has been authorized by employees of Brookhaven Science Associates, LLC under Contract No. DE-SC0012704 with the U.S. Department of Energy. The United States Government retains a nonexclusive, paid-up, irrevocable, world-wide license to publish or reproduce the published form of this document, or allow others to do so, for United States Government purposes. 


\title{
Booster Double Harmonic Setup Notes
}

\author{
C.J. Gardner
}

February 17, 2015

\section{Motivation and Setup Scenario}

1. Reducing the peak beam current reduces the transverse space charge force, which reduces beam loss due to resonance excitation. This reduction can be achieved by flattening the RF bucket with a second RF harmonic $[1,2,3]$.

2. Flattening the RF bucket also reduces the momentum spread of the beam, which reduces tune spread due to nonzero chromaticity and can reduce beam loss in regions of nonzero dispersion.

3. $\mathrm{RF}$ capture and acceleration of polarized protons $(\mathrm{PP})$ is first set up in the single harmonic mode with $\mathrm{RF}$ harmonic $h=1$. PP are adiabatically captured at injection with $h f=841.166738 \mathrm{kHz}$ (which corresponds to $200 \mathrm{MeV}$ kinetic energy) and then accelerated to $1362.77885 \mathrm{kHz}$ (which corresponds to $G_{p} \gamma=4.5$ ).

4. This is normally done with RF stations A3 and B3, but here we use stations A6 and E6, so that A3 and B3 (which have a greater frequency range) can be used to introduce the second harmonic for the double harmonic setup.

5. Once capture and acceleration have been set up in the single harmonic mode, the second harmonic system is brought on (with stations A3 and B3) and programmed to operate in concert with the single harmonic system.

6. The second harmonic system operates at harmonic $2 h=2$ with capture at $2 h f=1.682 \mathrm{MHz}$ and acceleration to $2.726 \mathrm{MHz}$.

7. The phase and amplitude of the second harmonic are programmed to produce a flattened RF bucket throughout the magnetic cycle. 


\section{Equations for Turn-by-Turn Motion}

The turn-by-turn motion for the double harmonic setup is governed by the potential $U$, where [4]

$$
\begin{gathered}
\frac{\partial U}{\partial \phi}=-F\left(\phi+\phi_{s}\right)+F\left(\phi_{s}\right) \\
F(\theta)=A_{1} \sin \theta-A_{2} \sin \left(2 \theta-2 \psi_{0}\right) .
\end{gathered}
$$

and

$$
A_{1}=\frac{e Q V_{1}}{2 \pi h}, \quad A_{2}=\frac{e Q V_{2}}{2 \pi h} .
$$

Here $\phi_{s}$ is the stable synchronous phase and $\phi$ is the deviation from $\phi_{s}$.

$\psi_{0}$ is an adjustable phase offset.

$e$ is the proton charge and $e Q$ is the charge of the particle.

$h$ is the RF harmonic number for the single harmonic setup.

$V_{1}$ and $V_{2}$ are adjustable amplitudes of the first and second harmonic voltages.

Thus we have

$$
\begin{gathered}
\frac{\partial U}{\partial \phi}=-A_{1} \sin \left(\phi+\phi_{s}\right)+A_{2} \sin \left(2 \phi+2 \phi_{s}-2 \psi_{0}\right)+C \\
\frac{\partial^{2} U}{\partial^{2} \phi}=-A_{1} \cos \left(\phi+\phi_{s}\right)+2 A_{2} \cos \left(2 \phi+2 \phi_{s}-2 \psi_{0}\right)
\end{gathered}
$$

and

$$
U(\phi)=A_{1} \cos \left(\phi+\phi_{s}\right)-\frac{1}{2} A_{2} \cos \left(2 \phi+2 \phi_{s}-2 \psi_{0}\right)+C \phi+D
$$

where

$$
C=A_{1} \sin \phi_{s}-A_{2} \sin \left(2 \phi_{s}-2 \psi_{0}\right)
$$

and $D$ is a constant independent of $\phi$.

If we define

$$
\psi=\phi+\phi_{s}
$$

then the potential $U$ expressed as a function of $\psi$ is

$$
U(\psi)=A_{1} \cos \psi-\frac{1}{2} A_{2} \cos \left(2 \psi-2 \psi_{0}\right)+C\left(\psi-\phi_{s}\right)+D .
$$


Choosing

$$
D=C \phi_{s}
$$

we then have

$$
\begin{gathered}
U(\psi)=A_{1} \cos \psi-\frac{1}{2} A_{2} \cos \left(2 \psi-2 \psi_{0}\right)+C \psi \\
\frac{\partial U}{\partial \psi}=-A_{1} \sin \psi+A_{2} \sin \left(2 \psi-2 \psi_{0}\right)+C
\end{gathered}
$$

and

$$
\frac{\partial^{2} U}{\partial^{2} \psi}=-A_{1} \cos \psi+2 A_{2} \cos \left(2 \psi-2 \psi_{0}\right)
$$

where

$$
C=A_{1} \sin \phi_{s}-A_{2} \sin \left(2 \phi_{s}-2 \psi_{0}\right)
$$

The turn-by-turn equations of motion are [4]

$$
\begin{gathered}
t_{n+1}=t_{n}+\frac{2 \pi}{\omega_{n}^{s}}\left(\frac{\omega_{n}^{s}-\omega_{n}}{\omega_{n}}\right) \\
e_{n+1}=e_{n}+e Q\left\{V\left(T_{n+1}^{s}+t_{n+1}\right)-V\left(T_{n+1}^{s}\right)\right\}
\end{gathered}
$$

where

$$
\begin{gathered}
V\left(T_{n+1}^{s}+t_{n+1}\right)=\left(\frac{2 \pi h}{e Q}\right) F\left(\phi_{n+1}+\phi_{n+1}^{s}\right) \\
V\left(T_{n+1}^{s}\right)=\left(\frac{2 \pi h}{e Q}\right) F\left(\phi_{n+1}^{s}\right)
\end{gathered}
$$

and

$$
\phi_{n}=t_{n} h \omega_{n}^{s}, \quad \phi_{n+1}=t_{n+1} h \omega_{n+1}^{s} .
$$

Here $t_{n}$ and $e_{n}$ are the time and energy deviations of a given particle (with respect to the arrival time and energy of the synchronous particle), and $\omega_{n}$ and $\omega_{n}^{s}$ are the angular frequencies of the particle and synchronous particle, respectively. In (17) and (18) the RF voltage $V$ is defined as an explicit function of time. We also define

$$
V\left(\phi_{n+1}+\phi_{n+1}^{s}\right)=\left(\frac{2 \pi h}{e Q}\right) F\left(\phi_{n+1}+\phi_{n+1}^{s}\right)
$$

and

$$
V\left(\phi_{n+1}^{s}\right)=\left(\frac{2 \pi h}{e Q}\right) F\left(\phi_{n+1}^{s}\right) .
$$




\section{$3 \quad$ RF Voltage}

As a function of phase, the RF voltage for the single harmonic setup is

$$
V(\psi)=V_{1} \sin \psi
$$

The synchronous phase $\phi_{1}$ is given by

$$
V_{1} \sin \phi_{1}=2 \pi R_{s} \rho_{s}\left(\frac{1}{c} \frac{d B}{d t}\right)
$$

where $R_{s}$ and $\rho_{s}$ are the radius and radius-of-curvature of the orbit

followed by the synchronous particle, and $B$ is the programmed guide field.

For the double harmonic setup the RF voltage is

$$
V(\psi)=V_{1} \sin \psi-V_{2} \sin \left(2 \psi-2 \psi_{0}\right) .
$$

The synchronous phase $\phi_{s}$ for this setup must satisfy

$$
V\left(\phi_{s}\right)=V_{1} \sin \phi_{1}
$$

where $V_{1} \sin \phi_{1}$ is given by (23). Thus we must have

$$
V_{1} \sin \phi_{s}-V_{2} \sin \left(2 \phi_{s}-2 \psi_{0}\right)=V_{1} \sin \phi_{1} .
$$

Using a prime to denote differentiation with respect to $\psi$ we have

$$
V^{\prime}\left(\phi_{s}\right)=V_{1} \cos \phi_{s}-2 V_{2} \cos \left(2 \phi_{s}-2 \psi_{0}\right)
$$

and

$$
V^{\prime \prime}\left(\phi_{s}\right)=-V_{1} \sin \phi_{s}+4 V_{2} \sin \left(2 \phi_{s}-2 \psi_{0}\right) .
$$

If we specify that

$$
V^{\prime}\left(\phi_{s}\right)=V_{1} F, \quad V^{\prime \prime}\left(\phi_{s}\right)=V_{1} G
$$

for some $F$ and $G$, then

$$
V_{1} \cos \phi_{s}-2 V_{2} \cos \left(2 \phi_{s}-2 \psi_{0}\right)=V_{1} F
$$

and

$$
-V_{1} \sin \phi_{s}+4 V_{2} \sin \left(2 \phi_{s}-2 \psi_{0}\right)=V_{1} G .
$$

Equations (26) and (31) can be written in matrix form as

$$
\left(\begin{array}{cc}
V_{1} & -V_{2} \\
-V_{1} & 4 V_{2}
\end{array}\right)\left(\begin{array}{c}
\sin \phi_{s} \\
\sin \left(2 \phi_{s}-2 \psi_{0}\right)
\end{array}\right)=\left(\begin{array}{c}
V_{1} \sin \phi_{1} \\
V_{1} G
\end{array}\right)
$$


from which we obtain

$$
3 V_{1} V_{2}\left(\begin{array}{c}
\sin \phi_{s} \\
\sin \left(2 \phi_{s}-2 \psi_{0}\right)
\end{array}\right)=\left(\begin{array}{rr}
4 V_{2} & V_{2} \\
V_{1} & V_{1}
\end{array}\right)\left(\begin{array}{c}
V_{1} \sin \phi_{1} \\
V_{1} G
\end{array}\right) .
$$

Thus

$$
3 \sin \phi_{s}=4 \sin \phi_{1}+G
$$

and

$$
3 V_{2} \sin \left(2 \phi_{s}-2 \psi_{0}\right)=V_{1}\left(\sin \phi_{1}+G\right) .
$$

From (30) we also have

$$
2 V_{2} \cos \left(2 \phi_{s}-2 \psi_{0}\right)=V_{1}\left(\cos \phi_{s}-F\right) .
$$

Dividing (35) by (36) then gives

$$
\tan \left(2 \phi_{s}-2 \psi_{0}\right)=\frac{2}{3}\left(\frac{\sin \phi_{1}+G}{\cos \phi_{s}-F}\right) .
$$

If $\phi_{1}$ and $G$ are given then $\phi_{s}$ can be obtained from equation (34). If $F$ is also given then equation (37) gives $\psi_{0}$ and equation (36) gives

$$
2 V_{2}=\frac{V_{1}\left(\cos \phi_{s}-F\right)}{\cos \left(2 \phi_{s}-2 \psi_{0}\right)} \text {. }
$$

Thus if we specify the synchronous phase $\phi_{1}$ for the single harmonic setup and if we choose values for the parameters $F$ and $G$, then equations (34), (37), and (38) give the synchronous phase $\phi_{s}$, the phase $\psi_{0}$, and the ratio $V_{2} / V_{1}$ for the double harmonic setup.

\section{Normalized Voltage, Potential, and RF Bucket}

We define normalized voltage

$$
\mathcal{V}(\psi)=\frac{1}{V_{1}} V(\psi)
$$

and normalized potential

$$
\mathcal{U}(\psi)=\frac{1}{A_{1}}\left\{U\left(\psi_{u}\right)-U(\psi)\right\}
$$

where

$$
V(\psi)=V_{1} \sin \psi-V_{2} \sin \left(2 \psi-2 \psi_{0}\right)
$$




$$
U(\psi)=A_{1} \cos \psi-\frac{1}{2} A_{2} \cos \left(2 \psi-2 \psi_{0}\right)+C \psi
$$

and

$$
C=A_{1} \sin \phi_{s}-A_{2} \sin \left(2 \phi_{s}-2 \psi_{0}\right) .
$$

The normalized RF bucket is defined by the curves

$$
\mathcal{B}(\psi)= \pm \sqrt{-\mathcal{U}(\psi)}
$$

The phase $\psi_{u}$ appearing in (40) is the unstable synchronous phase defined by

$$
V\left(\psi_{u}\right)=V\left(\phi_{s}\right)
$$

and (below transition)

$$
V^{\prime}\left(\psi_{u}\right)<0
$$

We then have

$$
\mathcal{U}^{\prime}=-\frac{1}{A_{1}} U^{\prime}(\psi)
$$

where

$$
\begin{aligned}
U^{\prime}(\psi)=-A_{1} & \sin \psi+A_{2} \sin \left(2 \psi-2 \psi_{0}\right)+C \\
-\frac{1}{A_{1}} U^{\prime}(\psi) & =\sin \psi-\frac{A_{2}}{A_{1}} \sin \left(2 \psi-2 \psi_{0}\right) \\
& -\sin \phi_{s}+\frac{A_{2}}{A_{1}} \sin \left(2 \phi_{s}-2 \psi_{0}\right) .
\end{aligned}
$$

With the help of (3) we have

$$
\frac{A_{2}}{A_{1}}=\frac{V_{2}}{V_{1}}
$$

which gives

$$
-\frac{1}{A_{1}} U^{\prime}(\psi)=\frac{1}{V_{1}} V(\psi)-\frac{1}{V_{1}} V\left(\phi_{s}\right)
$$

and

$$
\mathcal{U}^{\prime}(\psi)=\mathcal{V}(\psi)-\mathcal{V}\left(\phi_{s}\right)
$$

Thus

$$
\mathcal{U}^{\prime \prime}(\psi)=\mathcal{V}^{\prime}(\psi), \quad \mathcal{U}^{\prime \prime \prime}(\psi)=\mathcal{V}^{\prime \prime}(\psi), \quad \mathcal{U}^{\prime \prime \prime \prime}(\psi)=\mathcal{V}^{\prime \prime \prime}(\psi)
$$

where

$$
\mathcal{V}(\psi)=\sin \psi-\frac{V_{2}}{V_{1}} \sin \left(2 \psi-2 \psi_{0}\right)
$$




$$
\begin{gathered}
\mathcal{V}^{\prime}(\psi)=\cos \psi-\frac{2 V_{2}}{V_{1}} \cos \left(2 \psi-2 \psi_{0}\right) \\
\mathcal{V}^{\prime \prime}(\psi)=-\sin \psi+\frac{4 V_{2}}{V_{1}} \sin \left(2 \psi-2 \psi_{0}\right) \\
\mathcal{V}^{\prime \prime \prime}(\psi)=-\cos \psi+\frac{8 V_{2}}{V_{1}} \cos \left(2 \psi-2 \psi_{0}\right) .
\end{gathered}
$$

Equations (55) and (57) also give

$$
\mathcal{V}^{\prime \prime \prime}(\psi)+4 \mathcal{V}^{\prime}(\psi)=3 \cos \psi
$$

Thus at the stable synchronous phase $\phi_{s}$ we have

$$
\begin{gathered}
\mathcal{V}\left(\phi_{s}\right)=\sin \phi_{1}, \quad \mathcal{V}^{\prime}\left(\phi_{s}\right)=F, \quad \mathcal{V}^{\prime \prime}\left(\phi_{s}\right)=G \\
\mathcal{U}^{\prime}\left(\phi_{s}\right)=0, \quad \mathcal{U}^{\prime \prime}\left(\phi_{s}\right)=F, \quad \mathcal{U}^{\prime \prime \prime}\left(\phi_{s}\right)=G
\end{gathered}
$$

and

$$
\mathcal{U}^{\prime \prime \prime \prime}\left(\phi_{s}\right)=3 \cos \phi_{s}-4 F .
$$

At the unstable synchronous phase we have

$$
\mathcal{V}\left(\psi_{u}\right)=\mathcal{V}\left(\phi_{s}\right), \quad \mathcal{U}\left(\psi_{u}\right)=0, \quad \mathcal{U}^{\prime}\left(\psi_{u}\right)=0
$$

Expanding the normalized potential about the stable synchronous phase we have

$$
\begin{aligned}
\mathcal{U}(\psi) & =\mathcal{U}\left(\phi_{s}\right)+\frac{1}{2 !} F\left(\psi-\phi_{s}\right)^{2}+\frac{1}{3 !} G\left(\psi-\phi_{s}\right)^{3} \\
& +\frac{1}{4 !}\left(3 \cos \phi_{s}-4 F\right)\left(\psi-\phi_{s}\right)^{4}+\cdots
\end{aligned}
$$

If $F>0$ then $\mathcal{U}(\psi)$ reaches a minimum at $\psi=\phi_{s}$. We can make the minimum flat by keeping $F$ small and positive. This produces a flattened $\mathrm{RF}$ bucket. Note that if $G$ is nonzero then the minimum will not be centered in the potential well.

If $F=0$ and $G=0$ then

$$
\mathcal{U}(\psi)=\mathcal{U}\left(\phi_{s}\right)+\frac{1}{4 !}\left(3 \cos \phi_{s}\right)\left(\psi-\phi_{s}\right)^{4}+\cdots
$$

which also gives a flat minimum at $\psi=\phi_{s}$. In this case the minimum is centered in the potential well. 


\section{$5 \quad$ Flattened Bucket Option I}

Suppose we want to keep the synchronous phase the same for the single and double harmonic setups. This means that the second harmonic voltage must be zero at the synchronous phase $\phi_{1}$ for the single harmonic setup.

The RF system can then use the calculated value of $\phi_{1}$ to set the phase $\psi_{0}$ of the second harmonic voltage.

For easy reference we write down equations (34), (37), and (38) here as

$$
\begin{gathered}
3 \sin \phi_{s}=4 \sin \phi_{1}+G \\
\tan \left(2 \phi_{s}-2 \psi_{0}\right)=\frac{2}{3}\left(\frac{\sin \phi_{1}+G}{\cos \phi_{s}-F}\right) \\
2 V_{2} \cos \left(2 \phi_{s}-2 \psi_{0}\right)=V_{1}\left(\cos \phi_{s}-F\right) .
\end{gathered}
$$

Then we have

$$
\phi_{s}=\phi_{1}
$$

and it follows from (65), (66), and (67) that

$$
G=-\sin \phi_{1}, \quad \psi_{0}=\phi_{s}=\phi_{1}, \quad 2 V_{2}=V_{1}\left(\cos \phi_{s}-F\right) .
$$

For a flattened RF bucket we want $F$ to be small and positive. This gives

$$
2 V_{2}<V_{1} \cos \phi_{s}
$$

with $2 V_{2}$ close to but less than $V_{1} \cos \phi_{s}$. We call this Flattened Bucket Option I.

If we take single harmonic stable synchronous phase

$$
\phi_{1}=30^{\circ}
$$

then we have

$$
\phi_{s}=30^{\circ}, \quad \psi_{0}=30^{\circ}, \quad \frac{V_{2}}{V_{1}}<0.4330127
$$

Figure 1 shows the normalized potential and RF bucket for the case in which $V_{2} / V_{1}=0.41$.

Figure $\mathbf{2}$ is a magnified view of Figure $\mathbf{1}$ showing that the normalized potential does indeed reach a minimum at $\phi_{s}=30^{\circ}$. Note that the minimum is not centered in the potential well. 


\section{$6 \quad$ Flattened Bucket Option II}

If we want a flattened bucket with

$$
V^{\prime}\left(\phi_{s}\right)=0, \quad V^{\prime \prime}\left(\phi_{s}\right)=0
$$

then we have

$$
F=0, \quad G=0
$$

and it follows from (65), (66), and (67) that

$$
\begin{gathered}
\sin \phi_{s}=\frac{4}{3} \sin \phi_{1}, \quad \sin \phi_{1}=\frac{3}{4} \sin \phi_{s} \\
\tan \left(2 \phi_{s}-2 \psi_{0}\right)=\frac{1}{2} \tan \phi_{s}
\end{gathered}
$$

and

$$
2 V_{2}=\frac{V_{1} \cos \phi_{s}}{\cos \left(2 \phi_{s}-2 \psi_{0}\right)}
$$

These equations give

$$
\begin{gathered}
\phi_{s}=\arcsin \left\{\frac{4}{3} \sin \phi_{1}\right\} \\
\psi_{0}=\phi_{s}-\frac{1}{2} \arctan \left\{\frac{1}{2} \tan \phi_{s}\right\}
\end{gathered}
$$

and

$$
\frac{V_{2}}{V_{1}}=\frac{\cos \phi_{s}}{2 \cos \left(2 \phi_{s}-2 \psi_{0}\right)} .
$$

We call this Flattened Bucket Option II.

If we again take single harmonic stable synchronous phase

$$
\phi_{1}=30^{\circ}
$$

then we have

$$
\phi_{s}=41.8103^{\circ}, \quad \psi_{0}=29.7629^{\circ}, \quad V_{2} / V_{1}=0.408248 .
$$

Note that the value of $\psi_{0}$ obtained here is very close to the value obtained in the previous section for Flattened Bucket Option I.

Figure 3 shows the normalized potential and RF bucket for these numbers. Figure 4 is a magnified view of Figure $\mathbf{3}$ showing that the normalized potential does indeed reach a minimum at $\phi_{s}=41.8103^{\circ}$. Note that the minimum is now centered in the potential well. 
Figure 5 shows the potential wells of Figures $\mathbf{2}$ and $\mathbf{4}$ together for comparison. The stable synchronous phases are $30^{\circ}$ and $41.8103^{\circ}$, respectively. Although these are different, the centers of the two wells are very nearly the same.

\section{RF Capture}

During injection of PP in Booster the guide field is held constant and RF capture takes place by raising the $\mathrm{RF}$ voltage adiabatically from zero.

For capture with the single harmonic setup the stable synchronous phase is

$$
\phi_{1}=0 \text {. }
$$

Figure 6 shows the normalized voltage, potential, and RF bucket. Here

$$
\mathcal{V}(\psi)=\sin \psi
$$

and

$$
\mathcal{U}(\psi)=-\cos \psi-1 .
$$

For capture with the double harmonic setup we take

$$
\phi_{s}=\phi_{1}=0 .
$$

Equations (65), (66), and (67) then give

$$
G=0, \quad \psi_{0}=0, \quad 2 V_{2}=V_{1}(1-F) .
$$

Taking $F=0$ then gives the conditions for Flattened Bucket Option II.

Figure 7 shows the normalized harmonic 1 and 2 voltages and their sum

$$
\mathcal{V}(\psi)=\sin \psi-\frac{V_{2}}{V_{1}} \sin 2 \psi
$$

Here

$$
\begin{gathered}
V_{2} / V_{1}=1 / 2 \\
\mathcal{V}\left(\phi_{s}\right)=0, \quad \mathcal{V}^{\prime}\left(\phi_{s}\right)=0, \quad \mathcal{V}^{\prime \prime}\left(\phi_{s}\right)=0, \quad \mathcal{V}^{\prime \prime \prime}\left(\phi_{s}\right)=3 \\
\mathcal{U}^{\prime}\left(\phi_{s}\right)=0, \quad \mathcal{U}^{\prime \prime}\left(\phi_{s}\right)=0, \quad \mathcal{U}^{\prime \prime \prime}\left(\phi_{s}\right)=0, \quad \mathcal{U}^{\prime \prime \prime \prime}\left(\phi_{s}\right)=3
\end{gathered}
$$


and

$$
\mathcal{U}(\psi)=\mathcal{U}(0)+\frac{3}{4 !} \psi^{4}+\cdots .
$$

Figures 8 and $\mathbf{9}$ show the normalized potential and RF bucket.

Figures 10 and 11 show the normalized voltages, potentials, and RF buckets for single and double harmonic capture together for comparison.

Figure 12 shows the result of simulated single harmonic capture of PP in Booster at injection. The simulation starts with a uniform distribution of unbunched beam consisting of a 100-by-100 array of particles covering the rectangle indicated in the figure. The area of the distribution is $1 \mathrm{eV} \mathrm{s}$. The kinetic energy of the synchronous particle at the center of the distribution is $200 \mathrm{MeV}$. The RF voltage amplitude $V_{1}$ is raised quadratically from zero to $2 \mathrm{kV}$ over a time interval of $8 \mathrm{~ms}$.

Figure 13 shows the result of simulated double harmonic capture of PP at injection. The simulation starts with the same uniform distribution of unbunched beam as before. The RF voltage amplitude $V_{1}$ is again raised quadratically from zero to $2 \mathrm{kV}$ over a time interval of $8 \mathrm{~ms}$, but now the second harmonic voltage follows along with amplitude $V_{2}=V_{1} / 2$.

Figure 14 shows the two simulation results together for comparison. The bunch obtained with double harmonic capture is noticeably flatter. This effect becomes much more pronounced with acceleration.

\section{Acceleration}

During acceleration of PP in Booster with the single harmonic setup, the stable synchronous phase increases from zero at capture to a maximum of

$$
\phi_{1}=30^{\circ} .
$$

For Flattened Bucket Option I with $\phi_{1}=30$ degrees we have

$$
\phi_{s}=30^{\circ}, \quad \psi_{0}=30^{\circ}, \quad V_{2} / V_{1}<0.4330127
$$

and for Flattened Bucket Option II

$$
\phi_{s}=41.8103^{\circ}, \quad \psi_{0}=29.7629^{\circ}, \quad V_{2} / V_{1}=0.408248 .
$$

Figure 15 shows the normalized voltage, potential, and RF bucket for single harmonic acceleration of PP in Booster with stable synchronous phase $\phi_{1}=30^{\circ}$. 
Figures 16, 17, and 18 show the normalized voltages, potential, and RF bucket for double harmonic acceleration of PP in Booster with Flattened Bucket Option I. Here $\phi_{s}=\phi_{1}=30^{\circ}, \psi_{0}=30^{\circ}$, and $V_{2} / V_{1}=0.41$, just as in Figures 1 and 2.

Figures 19 and $\mathbf{2 0}$ show the normalized voltages, potentials, and RF buckets of Figures $\mathbf{1 5}$ and $\mathbf{1 8}$ together for comparison. One can see by inspection that for a stable synchronous phase of 30 degrees, the double harmonic bucket area is larger than that of the single harmonic bucket.

Figure 21 gives the same comparison for a stable synchronous phase of 35 degrees. For the double harmonic setup, Flattened Bucket Option I has been used with $\phi_{s}=\phi_{1}=35^{\circ}, \psi_{0}=35^{\circ}$, and $V_{2} / V_{1}=0.384$. One can see by inspection that the double harmonic bucket area is slightly smaller than that of the single harmonic bucket.

Figure 22 gives the comparison for a stable synchronous phase of 40 degrees. For the double harmonic setup, Flattened Bucket Option I has been used with $\phi_{s}=\phi_{1}=40^{\circ}, \psi_{0}=40^{\circ}$, and $V_{2} / V_{1}=0.347$. One can see by inspection that the double harmonic bucket area is significantly smaller than that of the single harmonic bucket. Thus for synchronous phases greater than 35 degrees, the double harmonic setup may not be particularly useful.

Figure 23 shows the result of simulated single harmonic acceleration of $\mathrm{PP}$ in Booster. Here a $1 \mathrm{eV}$ s uniform distribution of unbunched beam was captured as in Figure $\mathbf{1 2}$ and then accelerated to a synchronous proton kinetic energy of $386.8495 \mathrm{MeV}$. The corresponding revolution frequency is 1.04915084266 MHz. The stable synchronous phase is 30 degrees.

Figure 24 shows the result of simulated double harmonic acceleration using Flattened Bucket Option I. Here a $1 \mathrm{eV}$ s uniform distribution of unbunched beam was captured as in Figure $\mathbf{1 3}$ and again accelerated to a synchronous proton kinetic energy of $386.8495 \mathrm{MeV}$. The stable synchronous phase is again 30 degrees. Note that the bucket and bunch are not centered on the synchronous phase (which has time deviation zero in the figure), but the bunch is centered in the bucket. This is in accordance with Figure 5 and the findings of sections 5 and 6 .

Figure 25 shows the simulation results of Figures 23 and $\mathbf{2 4}$ together for comparison.

Figure 26 shows the binned data of Figure 25. Here one sees that the double harmonic setup reduces the peak current by about $30 \%$. 
Figure 27 gives a comparison of the results of simulated single and double harmonic acceleration, but now with Flattened Bucket Option II used for the double harmonic setup. The synchronous proton kinetic energy is again $386.8495 \mathrm{MeV}$. The stable synchonous phase for the double harmonic setup is 41.8103 degrees. Note that the flattened bucket and bunch are now centered on the synchronous phase (which has time deviation zero in the figure). This is again in accordance with Figure $\mathbf{5}$ and the findings of sections 5 and 6 .

Figure 28 shows the binned data of Figure 27. Here again one sees that the double harmonic setup reduces the peak current by about $30 \%$.

\section{Implementation}

The Booster RF system was set up as outlined in section 1. Flattened Bucket Option I was adopted as the simplest way to move from the single to the double harmonic setup. Freddy Severino [5] and Kevin Smith made the necessary changes to the RF system. In particular, they used the calculated synchronous phase $\phi_{1}$ for the single harmonic setup to automatically set the phase $\psi_{0}$ of the second harmonic voltage. With the help of a mountain range display of the circulating bunched beam, the amplitude $V_{2}$ was then programmed by Keith Zeno to produce flattened bunches. The end result was significantly reduced beam loss during early acceleration where the space charge force is largest $[6,7,8,9]$. The double harmonic setup has been found to be robust in that once it has been set up, it requires minimal further tuning.

In the future we may want to consider using Flattened Bucket Option II. This would allow the second harmonic voltage $V_{2}$ to be programmed automatically. From the synchronous phase $\phi_{1}$ of the single harmonic setup one would use equations (78), (79), and (80) to calculate $\phi_{s}, \psi_{0}$, and $V_{2}$.

Figures 29 and $\mathbf{3 0}$ show the values of $\phi_{s}, \psi_{0}$, and $V_{2} / V_{1}$ obtained as $\phi_{1}$ varies from 0 to 30 degrees.

Figure 31 shows $\phi_{1}, \phi_{s}, \psi_{0}$, and $V_{2} / V_{1}$ along with the guide field $B$ and $d B / d t$ for capture of $\mathrm{PP}$ at $200 \mathrm{MeV}$ and acceleration to $386.8495 \mathrm{MeV}$ (kinetic energy) using Flattened Bucket Option II. 


\section{References}

[1] A. Hofmann and S. Myers, "Beam Dynamics in a Double RF System", CERN ISR-TH-RF/80-26, July 1980

[2] J.M. Baillod et al., "A Second Harmonic (6-16 MHz) RF System with Feedback-Reduced Gap Impedance for Accelerating Flat-Topped Bunches in the CERN PS Booster", IEEE Trans. Nucl. Sci. NS-30, 3499 (1983)

[3] J.M. Brennan et al., "High Intensity Performance of the Brookhaven AGS", Proceedings of the 1999 Particle Accelerator Conference, 614 (1999)

[4] C.J. Gardner, "Simulations of Bunch Merging in Booster", C-A/AP Note 176, October 2004, pp. 12-22.

[5] F.S. Severino, Booster-RF-stay elog, entries 16:43 through 17:15, January 15, 2015

[6] K.L. Zeno, Booster-AGS-pp-2015 elog, entry 17:24 January 15, 2015

[7] K.L. Zeno, Booster-AGS-pp-2015 elog, entry 17:09 January 23, 2015

[8] K.L. Zeno, Booster-AGS-pp-2015 elog, entry 17:38 January 23, 2015

[9] K.L. Zeno, Booster-AGS-pp-2015 elog, entry 14:16 January 29, 2015 


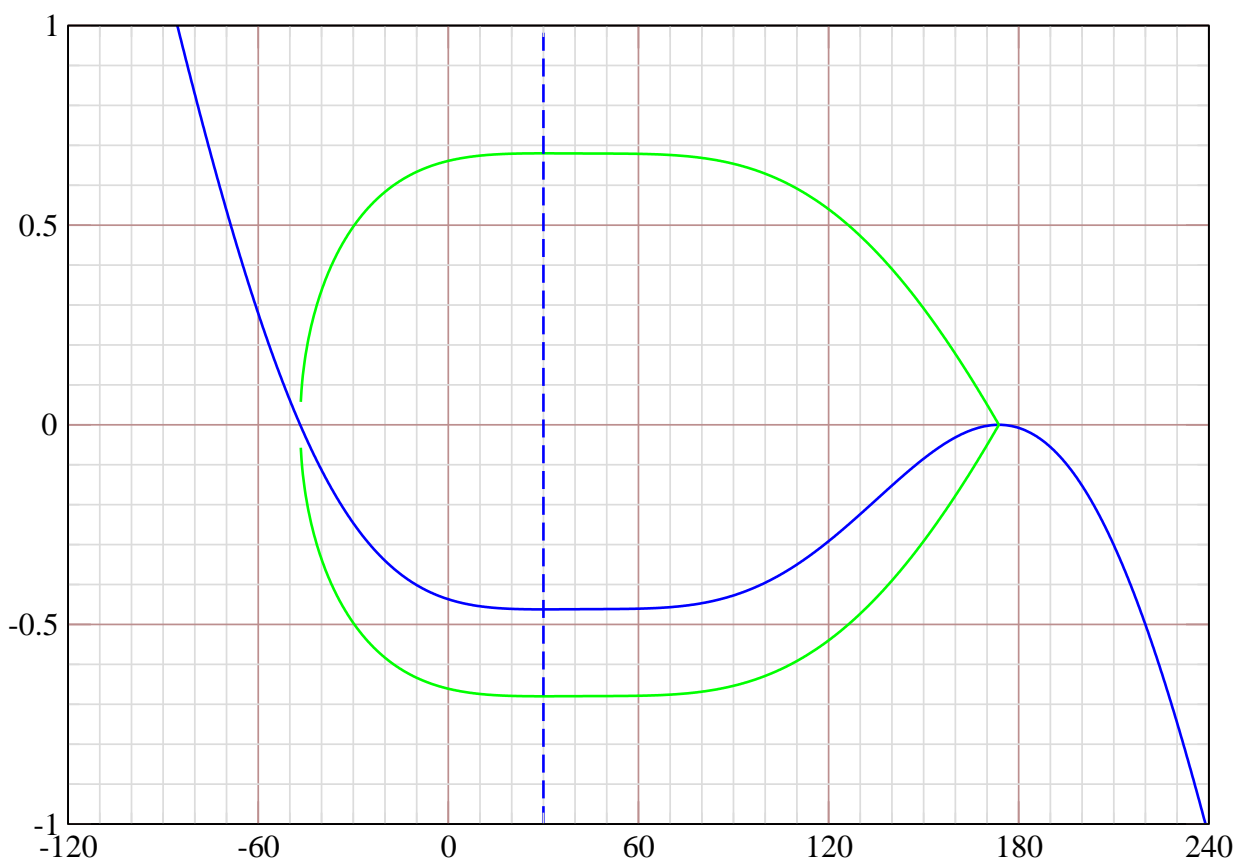

Figure 1: Normalized potential (blue curve) and RF bucket (green curve) for Flattened Bucket Option I. Here $\phi_{s}=\phi_{1}=30^{\circ}, \psi_{0}=30^{\circ}$, and $V_{2} / V_{2}=0.41$. The dashed blue line marks the stable synchronous phase $\phi_{s}$. 


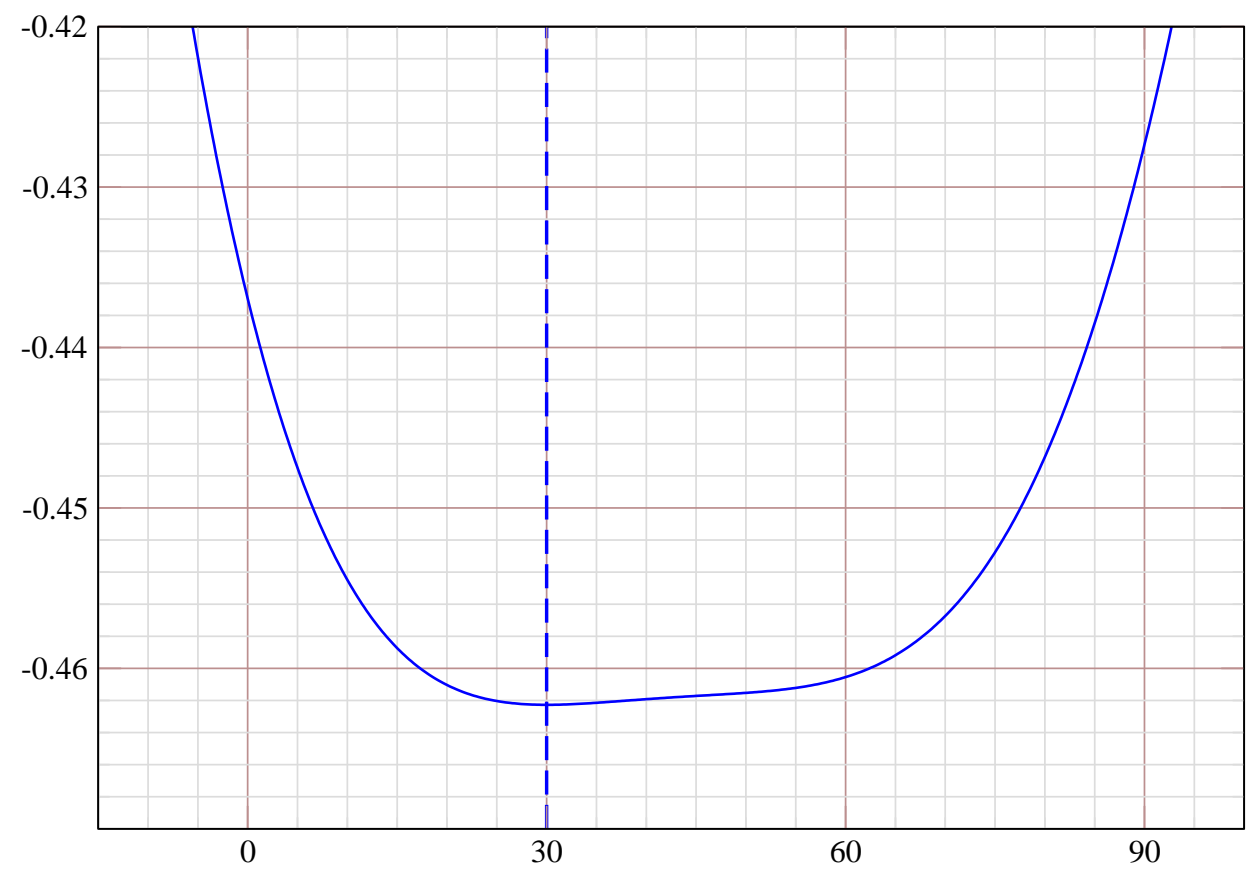

Figure 2: Magnified view of Figure 1 showing that the normalized potential for Flattened Bucket Option I does indeed reach a minimum at $\phi_{s}=30^{\circ}$. Note that the minimum is not centered in the potential well. 


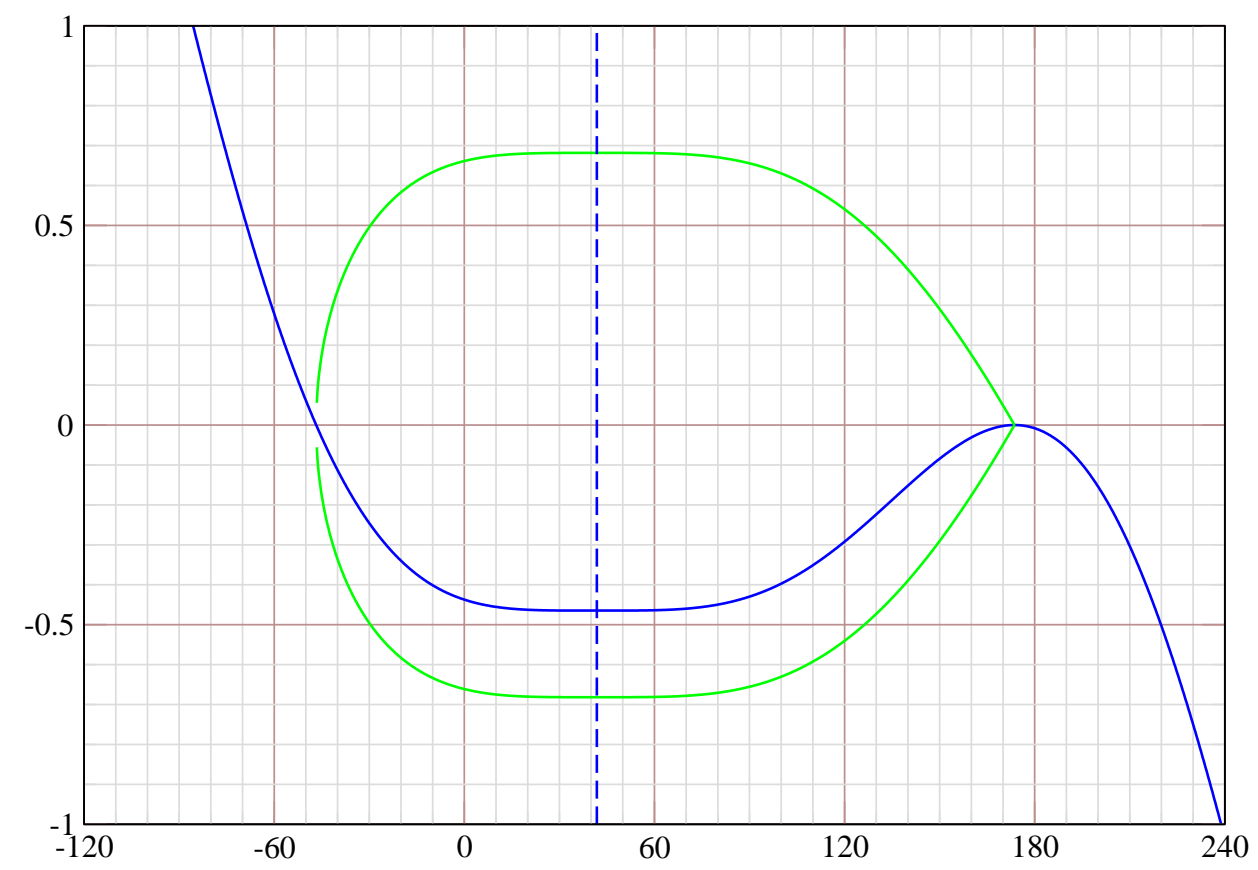

Figure 3: Normalized potential (blue curve) and RF bucket (green curve) for Flattened Bucket Option II. Here $\phi_{s}=41.8103^{\circ}, \psi_{0}=29.7629^{\circ}$, and $V_{2} / V_{1}=0.408248$. The dashed blue line marks the stable synchronous phase $\phi_{s}$. 


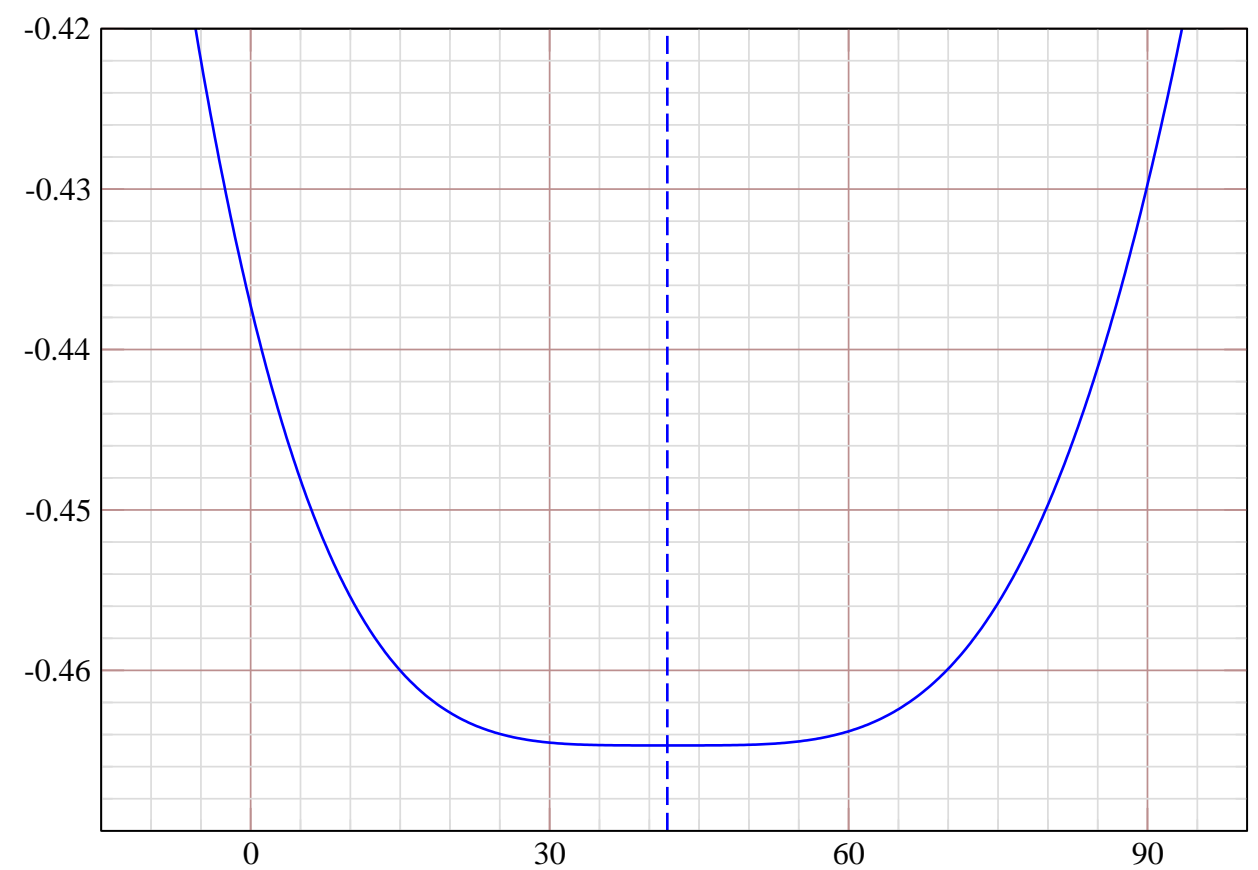

Figure 4: Magnified view of Figure 3 showing that the normalized potential for Flattened Bucket Option II does indeed reach a minimum at $\phi_{s}=41.8103^{\circ}$. Note that the minimum is now centered in the potential well. 


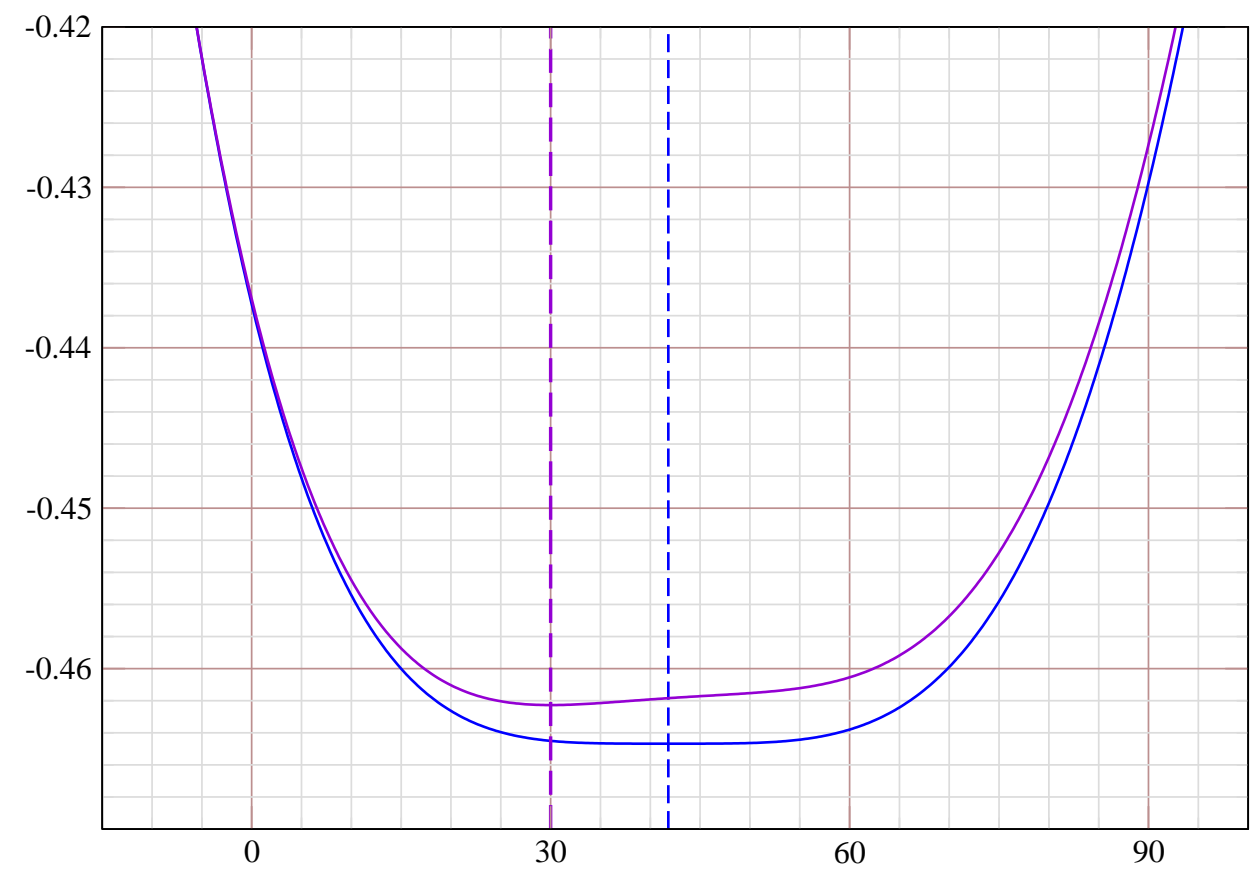

Figure 5: Here the potential wells of Figures $\mathbf{2}$ and $\mathbf{4}$ are shown together for comparison. The corresponding stable synchronous phases are $30^{\circ}$ and $41.8103^{\circ}$, respectively. Although these are different, the centers of the two wells are very nearly the same. 


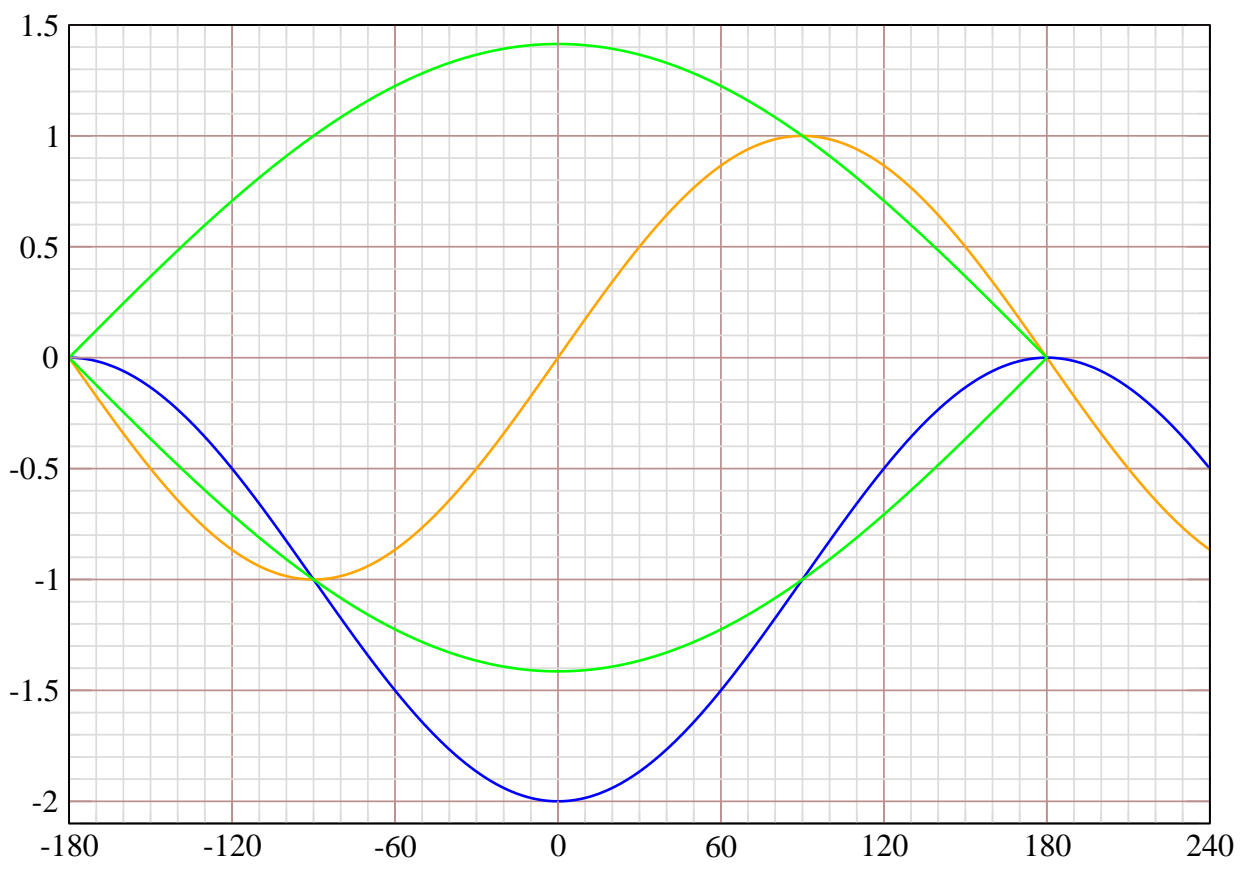

Figure 6: Normalized voltage, potential, and RF bucket for single harmonic capture of $\mathrm{PP}$ at Booster injection. These are the orange, blue, and green curves respectively. The horizontal axis gives the phase $\psi$ in degrees. The voltage $V(\psi)=V_{1} \sin \psi$ goes from negative to positive as $\psi$ passes through the stable synchronous phase $\phi_{1}=0$. The normalized potential is $\mathcal{U}(\psi)=$ $-\cos \psi-1$. 


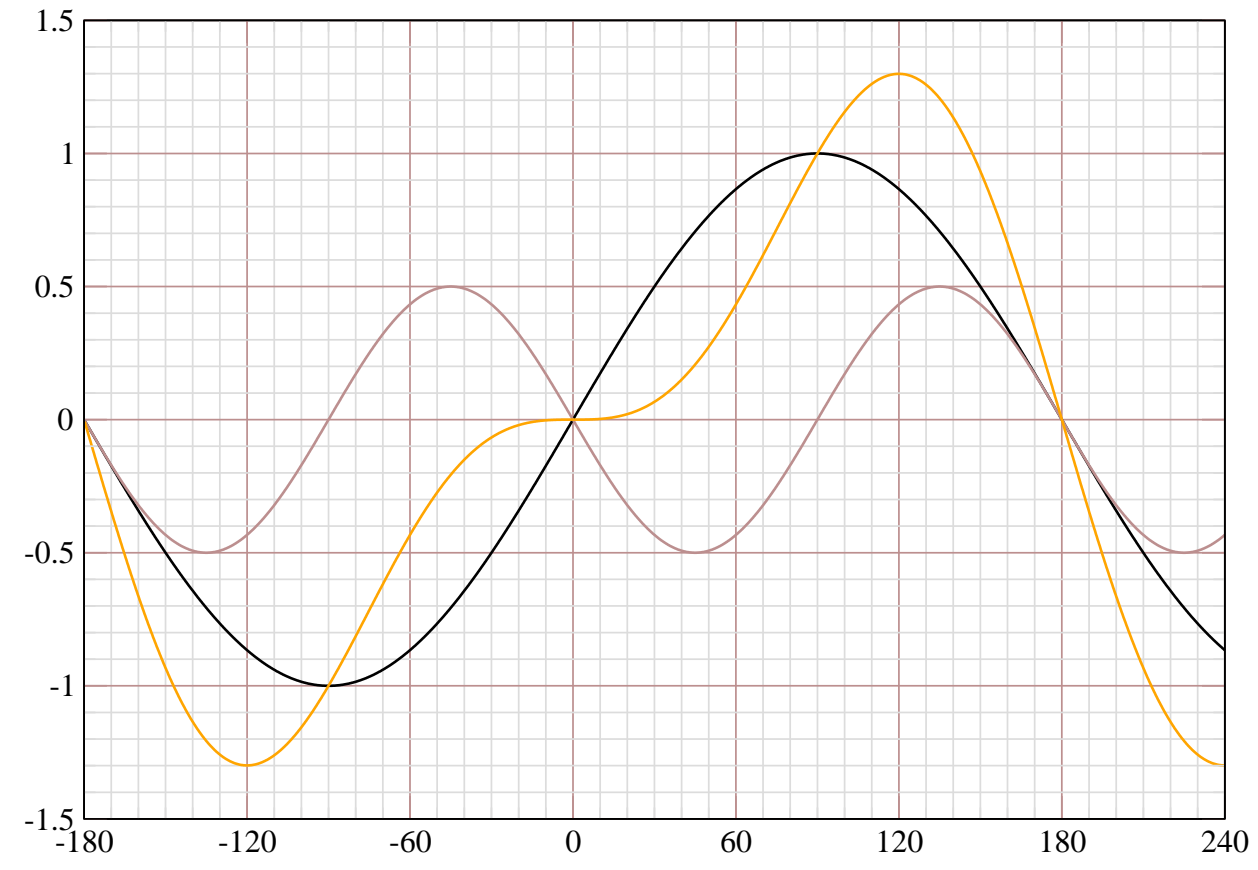

Figure 7: Normalized harmonic 1 and 2 voltages and their sum for double harmonic capture of PP at Booster injection. These are the black, brown, and orange curves, respectively. The horizontal axis gives the phase $\psi$ in degrees. The sum of the voltages is $V(\psi)=V_{1} \sin \psi-V_{2} \sin 2 \psi$. Note that the harmonic 2 voltage (brown curve) goes from positive to negative as the phase $\psi$ passes through the stable synchronous phase $\phi_{s}=0$. The ratio $V_{2} / V_{1}=1 / 2$ flattens the voltage sum at $\psi=\phi_{s}$. 


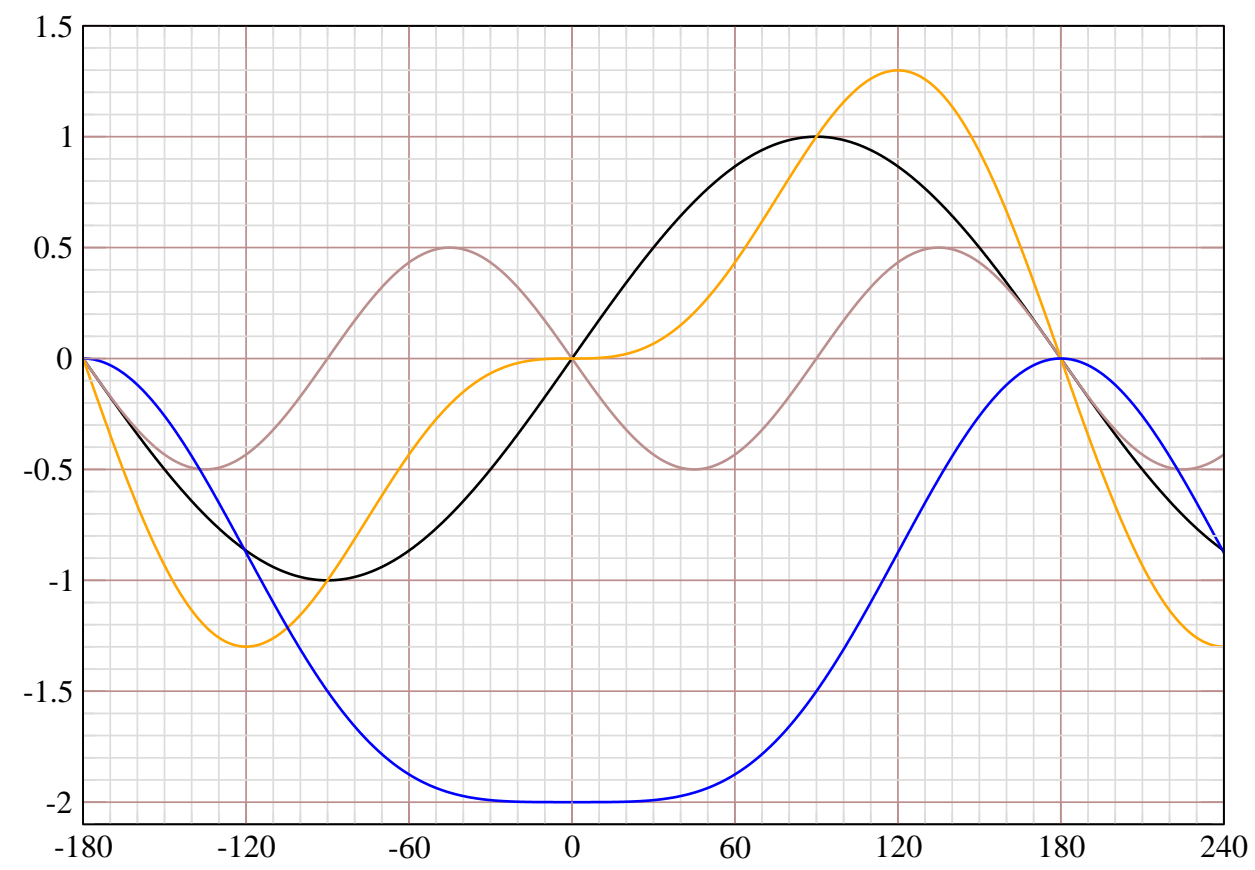

Figure 8: Normalized voltages and potential for double harmonic capture of $\mathrm{PP}$ at Booster injection. The horizontal axis gives the phase $\psi$ in degrees. The blue curve is the normalized potential. Its expansion about $\phi_{s}=0$ is given by $(92)$. 


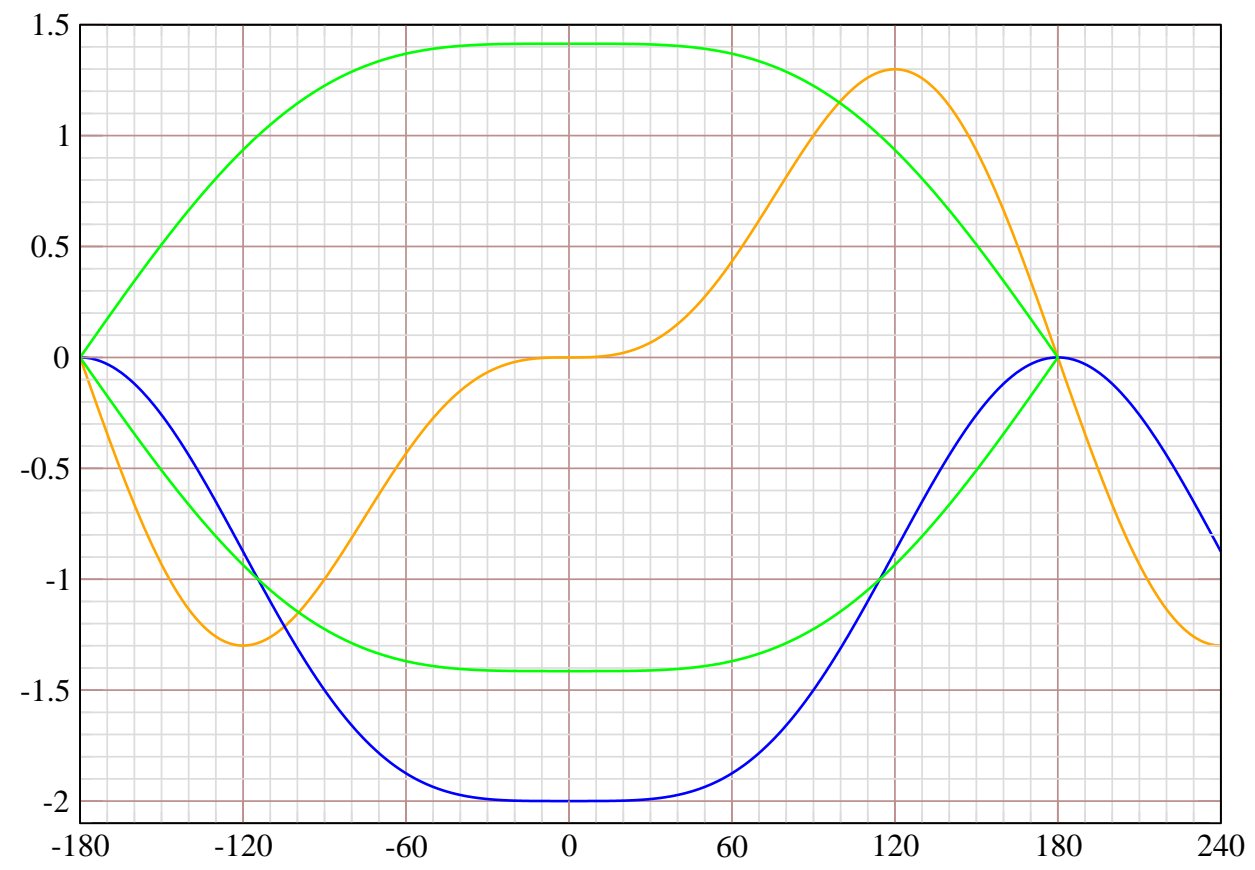

Figure 9: Normalized voltage, potential, and RF bucket for double harmonic capture of PP at Booster injection. The green curve is the RF bucket. 


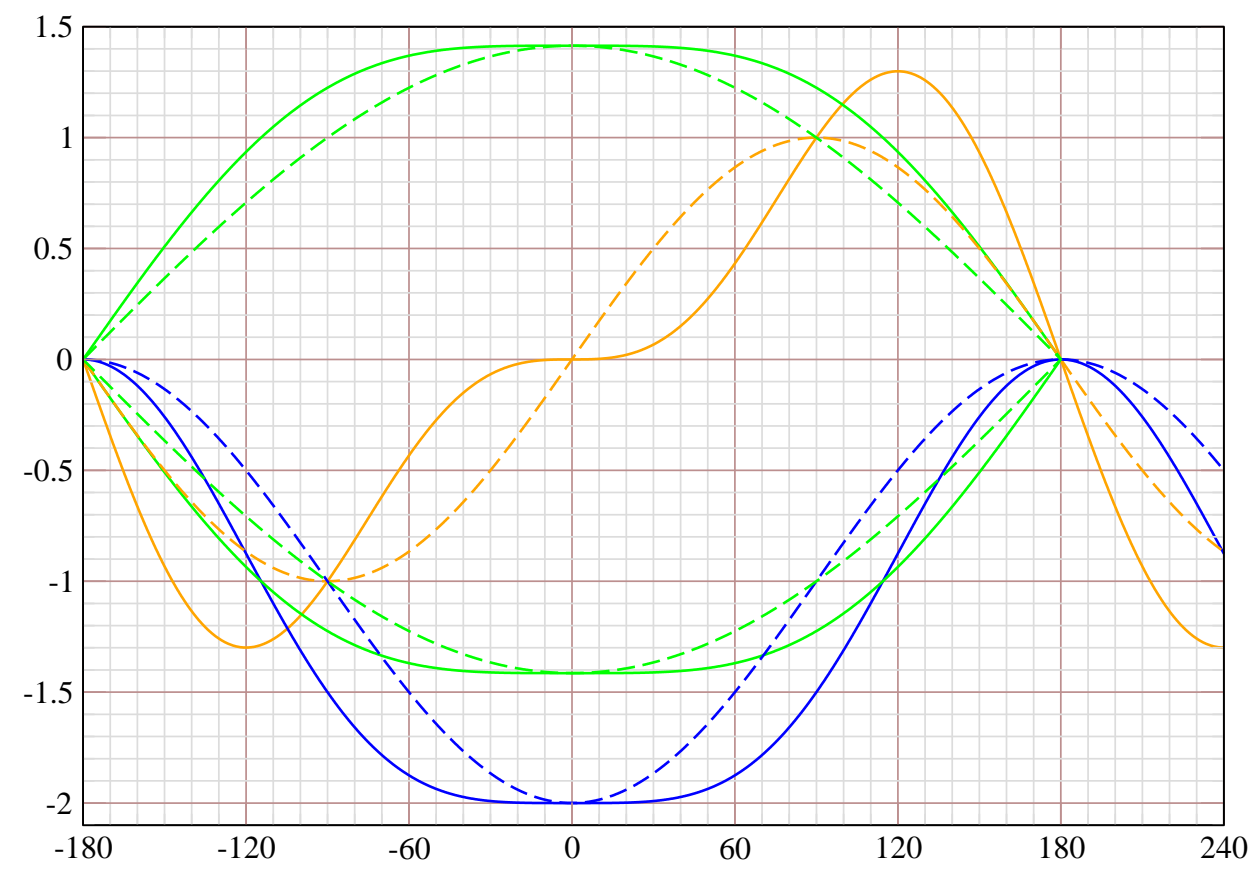

Figure 10: Here the normalized voltages, potentials, and RF buckets for single and double harmonic capture are shown together for comparison. 


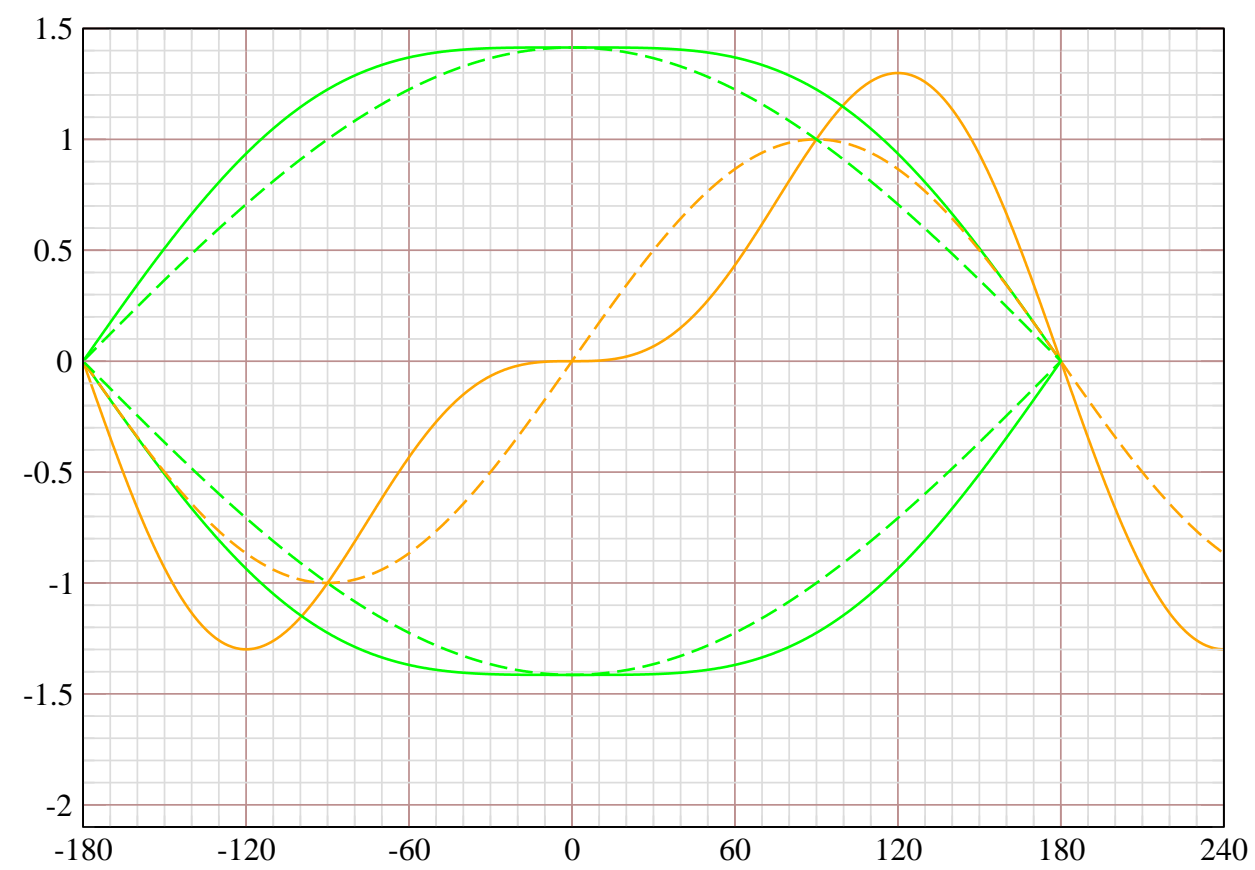

Figure 11: Same as Figure 10 but showing just the normalized voltages and RF buckets. 


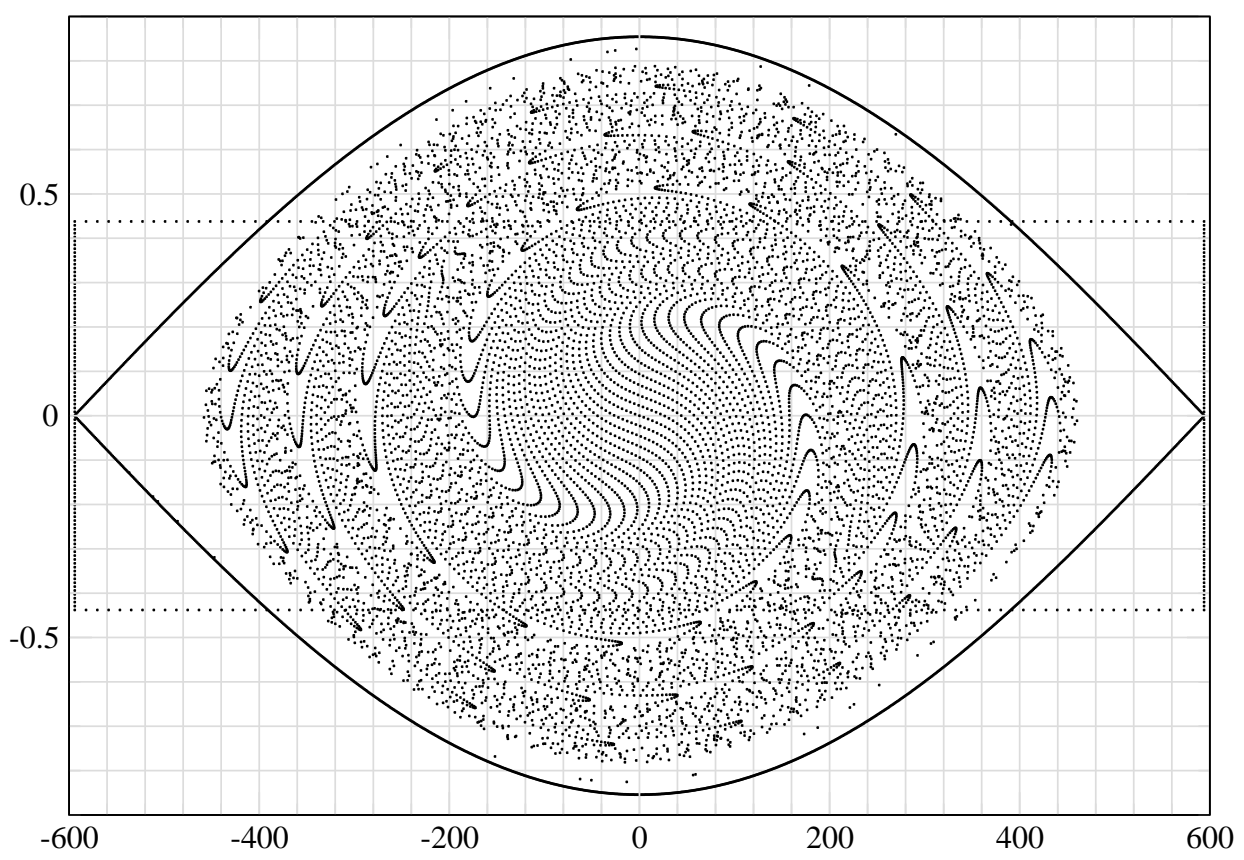

Figure 12: Result of simulated single harmonic capture of PP in Booster at injection. The simulation starts with a uniform distribution of unbunched beam consisting of a 100-by-100 array of particles covering the rectangle indicated in the figure. The area of the distribution is $1 \mathrm{eVs}$. The kinetic energy of the synchronous particle at the center of the distribution is 200 $\mathrm{MeV}$. The RF voltage amplitude $V_{1}$ is raised quadratically from zero to $2 \mathrm{kV}$ over a time interval of $8 \mathrm{~ms}$. The units of the horizontal and vertical axes are nanoseconds and $\mathrm{MeV}$ respectively. The synchronous revolution period is 1188.825 nanoseconds. 


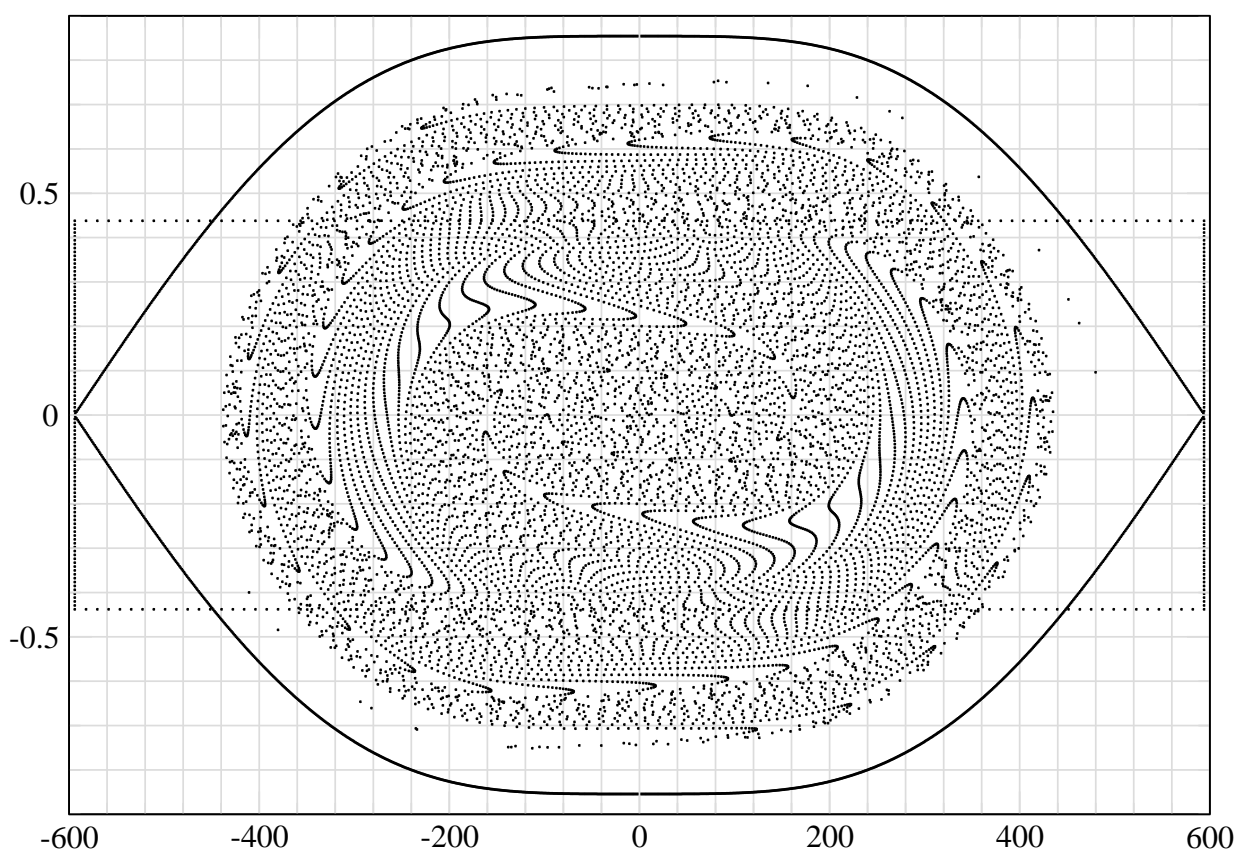

Figure 13: Result of simulated double harmonic capture of PP at injection. The simulation starts with the same uniform distribution of unbunched beam as before. The RF voltage amplitude $V_{1}$ is again raised quadratically from zero to $2 \mathrm{kV}$ over a time interval of $8 \mathrm{~ms}$, but now the second harmonic voltage follows along with amplitude $V_{2}=V_{1} / 2$. The units of the horizontal and vertical axes are again nanoseconds and $\mathrm{MeV}$. The synchronous revolution period is 1188.825 nanoseconds. 


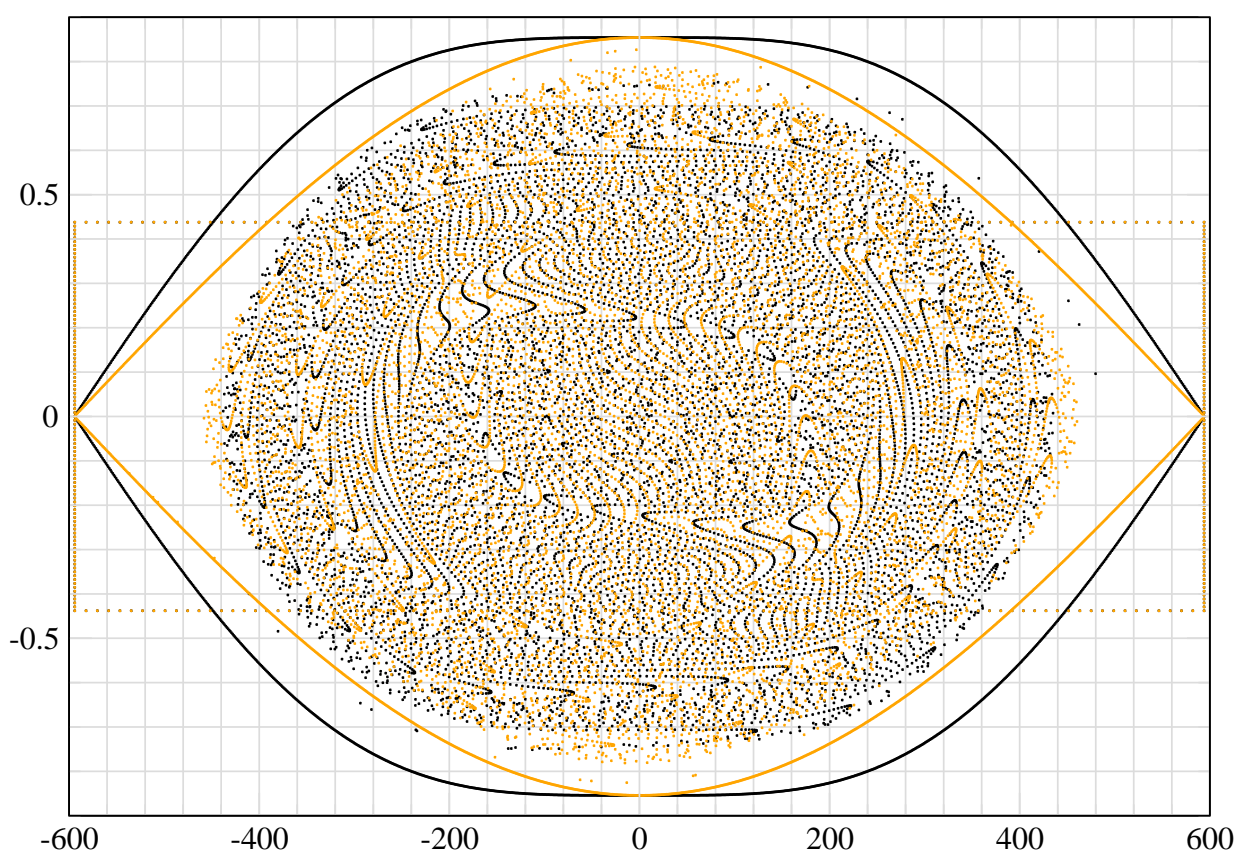

Figure 14: Here the results of simulated single and double harmonic capture of $\mathrm{PP}$ in Booster are shown together for comparison. The bunch obtained with double harmonic capture is noticeably flatter. This effect becomes much more pronounced with acceleration. 


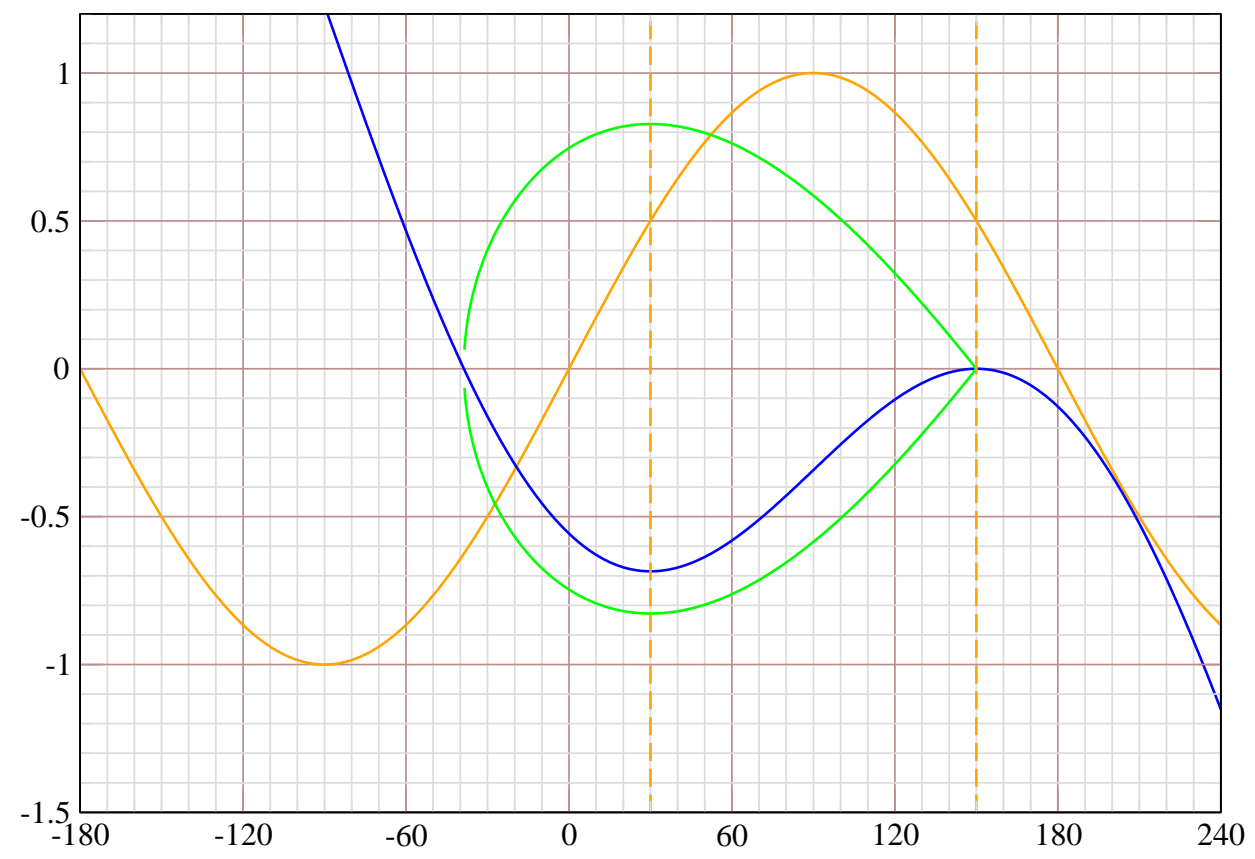

Figure 15: Normalized voltage, potential, and RF bucket for single harmonic acceleration in Booster. These are the orange, blue, and green curves respectively. The stable synchronous phase is 30 degrees. The stable and unstable synchronous phases are marked by the dashed vertical lines at 30 and 150 degrees respectively. 


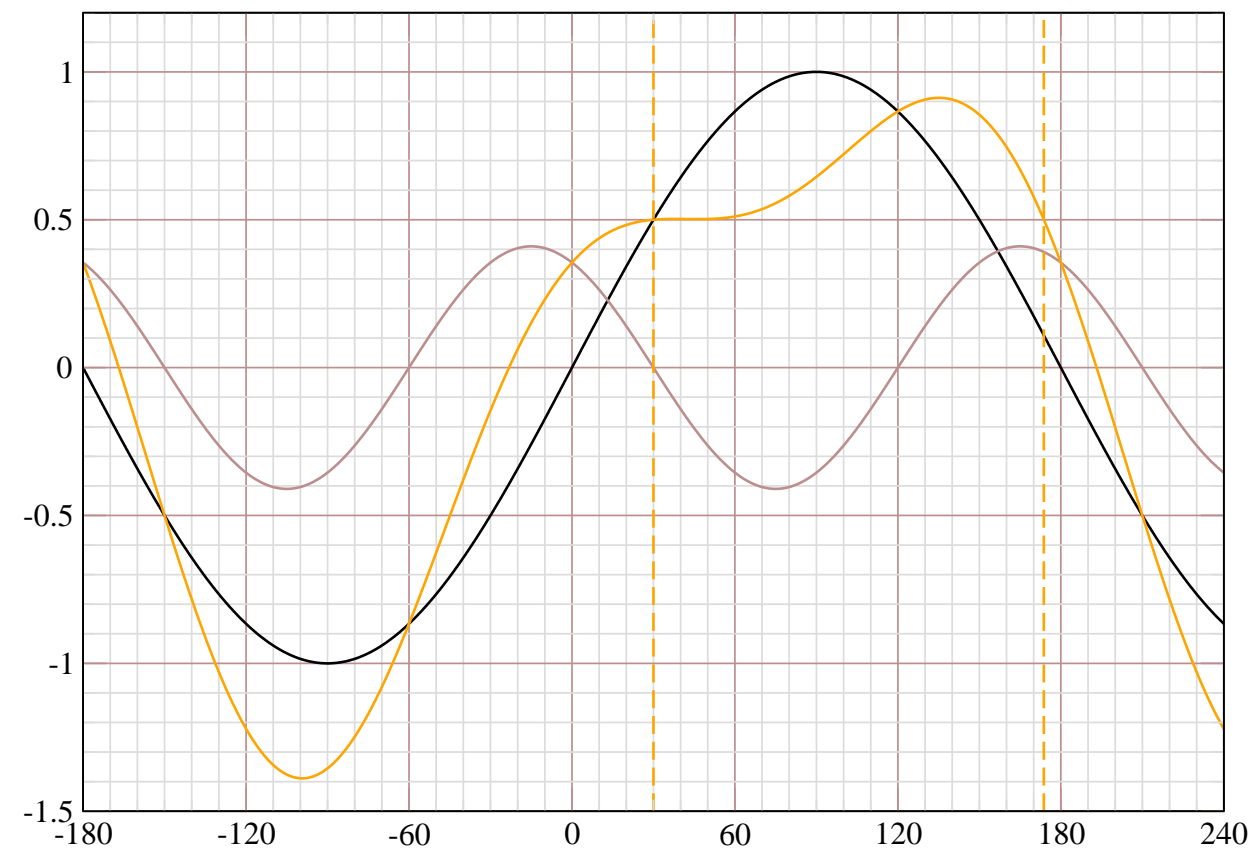

Figure 16: Normalized harmonic 1 and 2 voltages and their sum $\mathcal{V}(\psi)=$ $\sin \psi-\left(V_{2} / V_{1}\right) \sin \left(2 \psi-2 \phi_{s}\right)$ for double harmonic acceleration in Booster. These are the black, brown, and orange curves, respectively. The stable and unstable synchronous phases are marked by the dashed vertical lines at 30 and 173.745 degrees respectively. Here we have used Flattened Bucket Option I with $\phi_{s}=\phi_{1}=30^{\circ}, \psi_{0}=30^{\circ}$, and $V_{2} / V_{1}=0.41$, just as in Figures 1 and 2. Note that the harmonic 2 voltage (brown curve) goes from positive to negative and the sum of the voltages is flattened as the phase $\psi$ passes through the stable synchronous phase. 


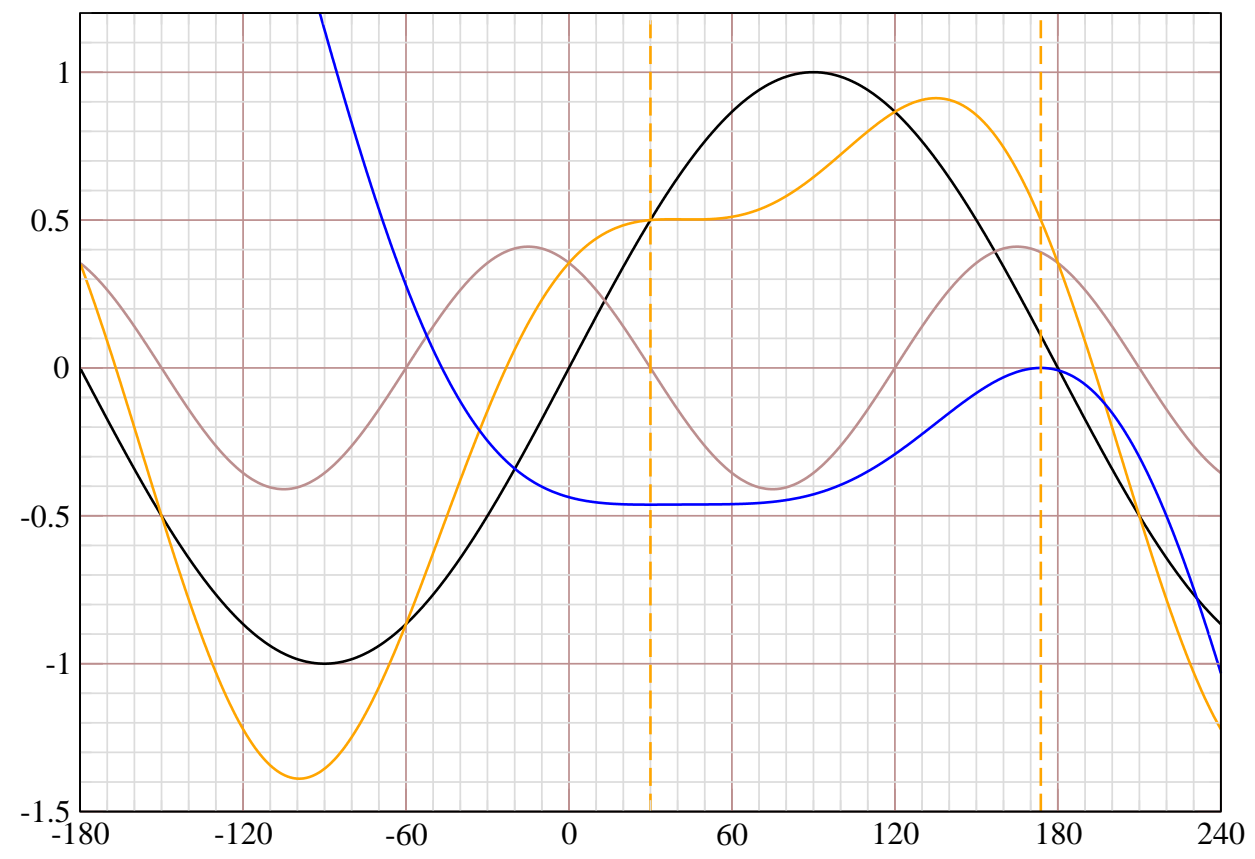

Figure 17: Same as Figure $\mathbf{1 6}$ but showing the normalized potential (blue curve). A magnified view of this potential is shown in Figure 2. 


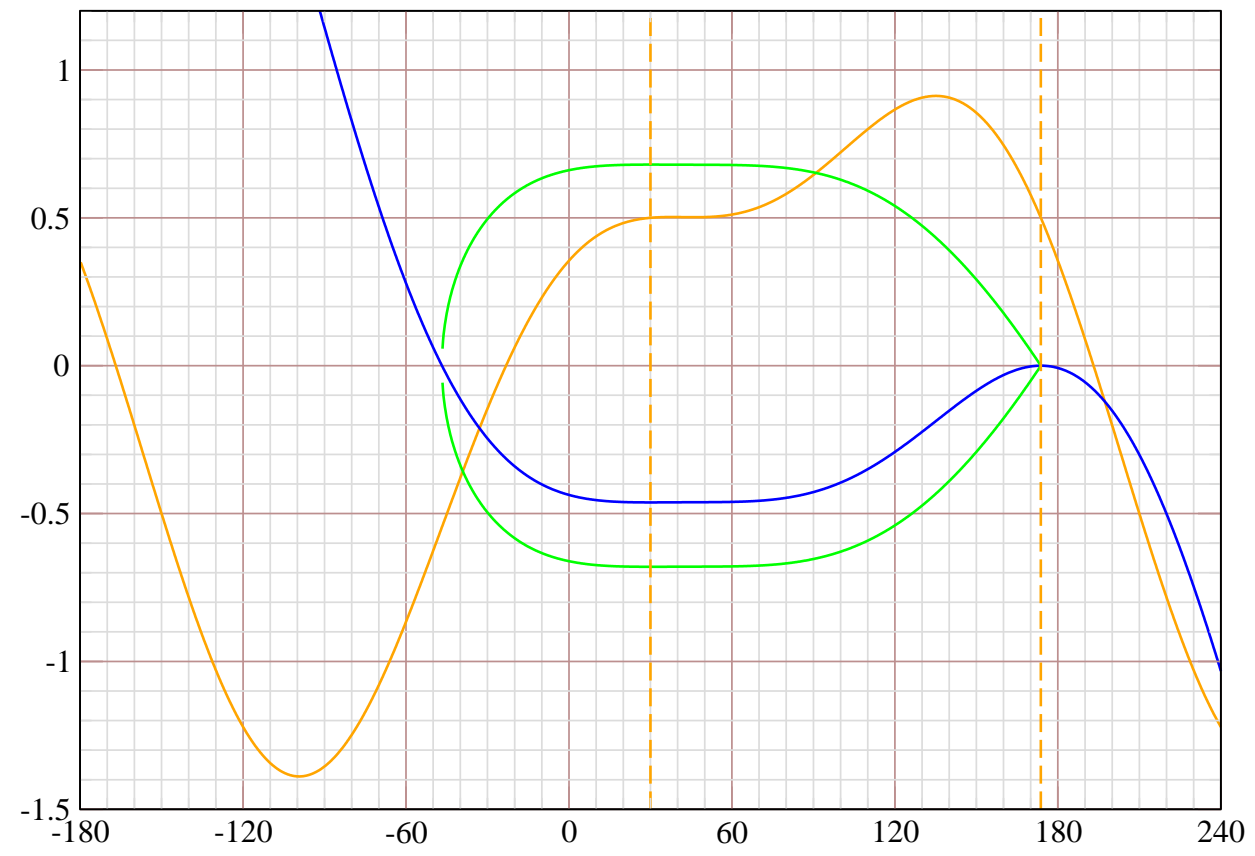

Figure 18: Same as Figure 17 but showing the RF bucket (green curve). 


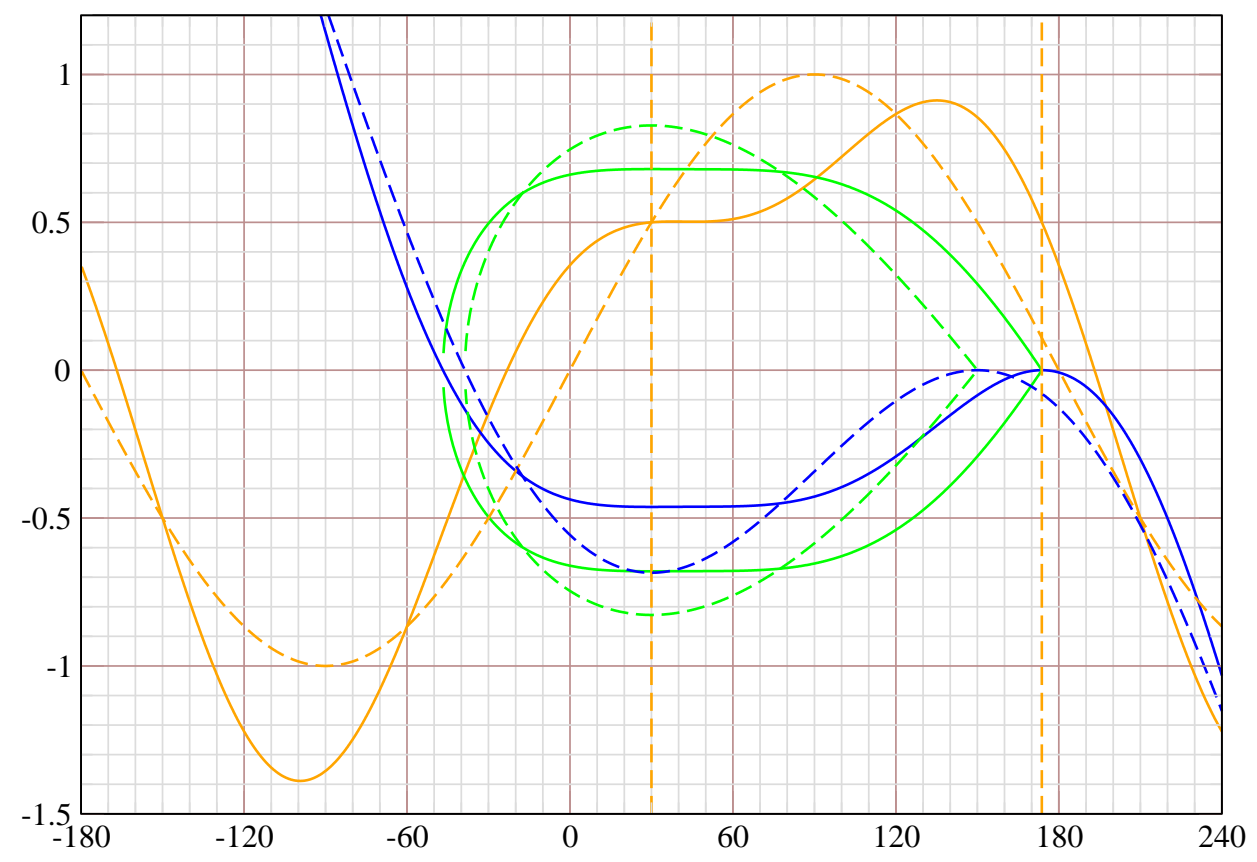

Figure 19: Here the normalized voltages, potentials, and RF buckets of Figures 15 and $\mathbf{1 8}$ are shown together for comparison. 


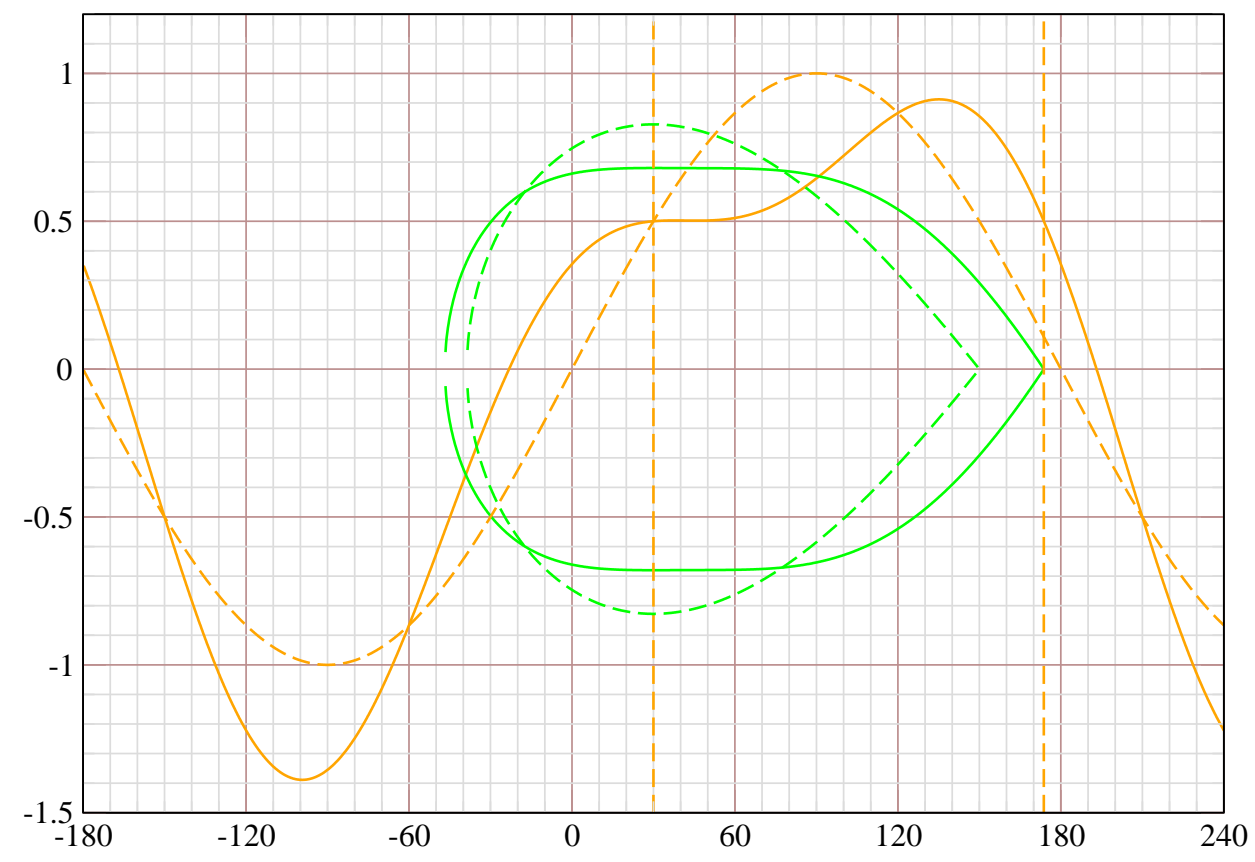

Figure 20: Same as Figure 19 but showing just the normalized voltages and RF buckets. One can see by inspection that for a stable synchronous phase of 30 degrees, the double harmonic bucket area is larger than that of the single harmonic bucket. 


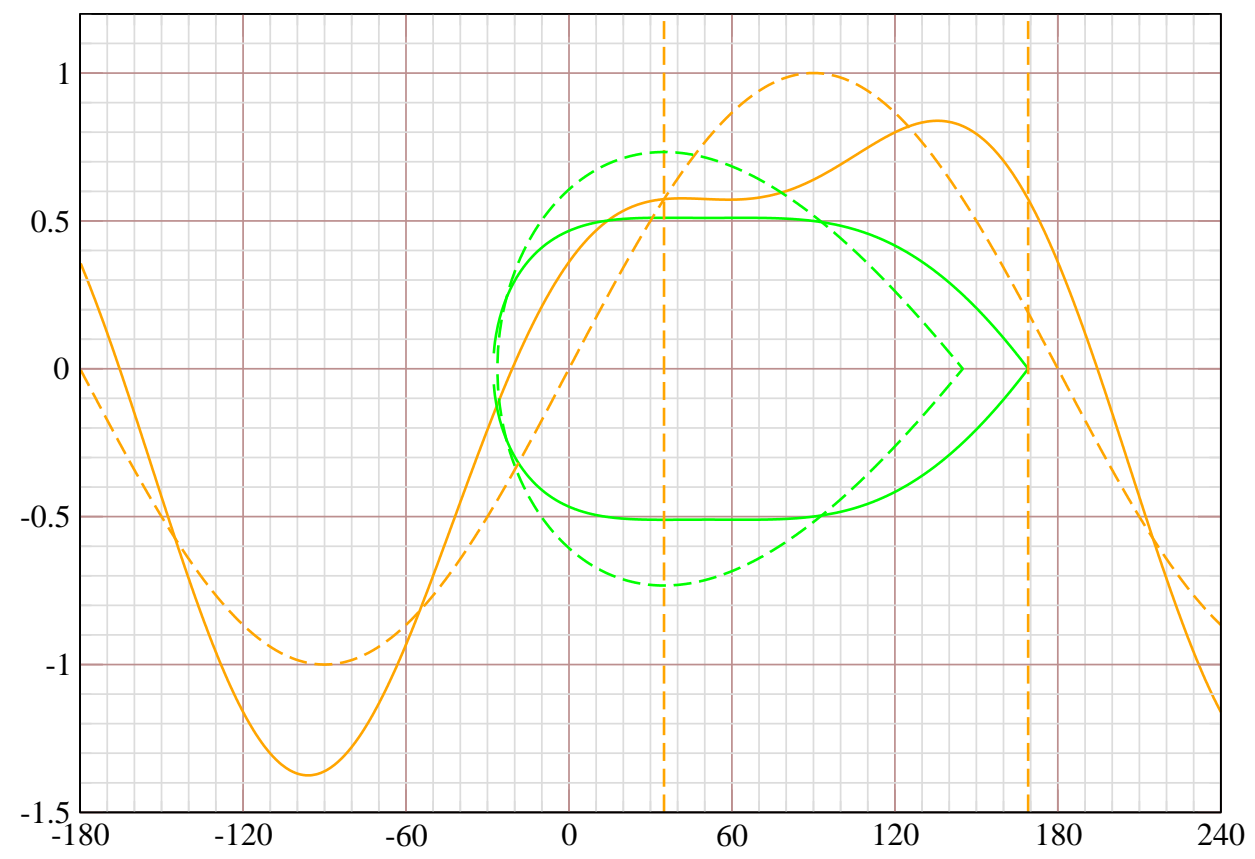

Figure 21: Same comparison but with a stable synchronous phase of 35 degrees. For the double harmonic setup, Flattened Bucket Option I has been used with $\phi_{s}=\phi_{1}=35^{\circ}, \psi_{0}=35^{\circ}$, and $V_{2} / V_{1}=0.384$. One can see by inspection that the double harmonic bucket area is slightly smaller than that of the single harmonic bucket. 


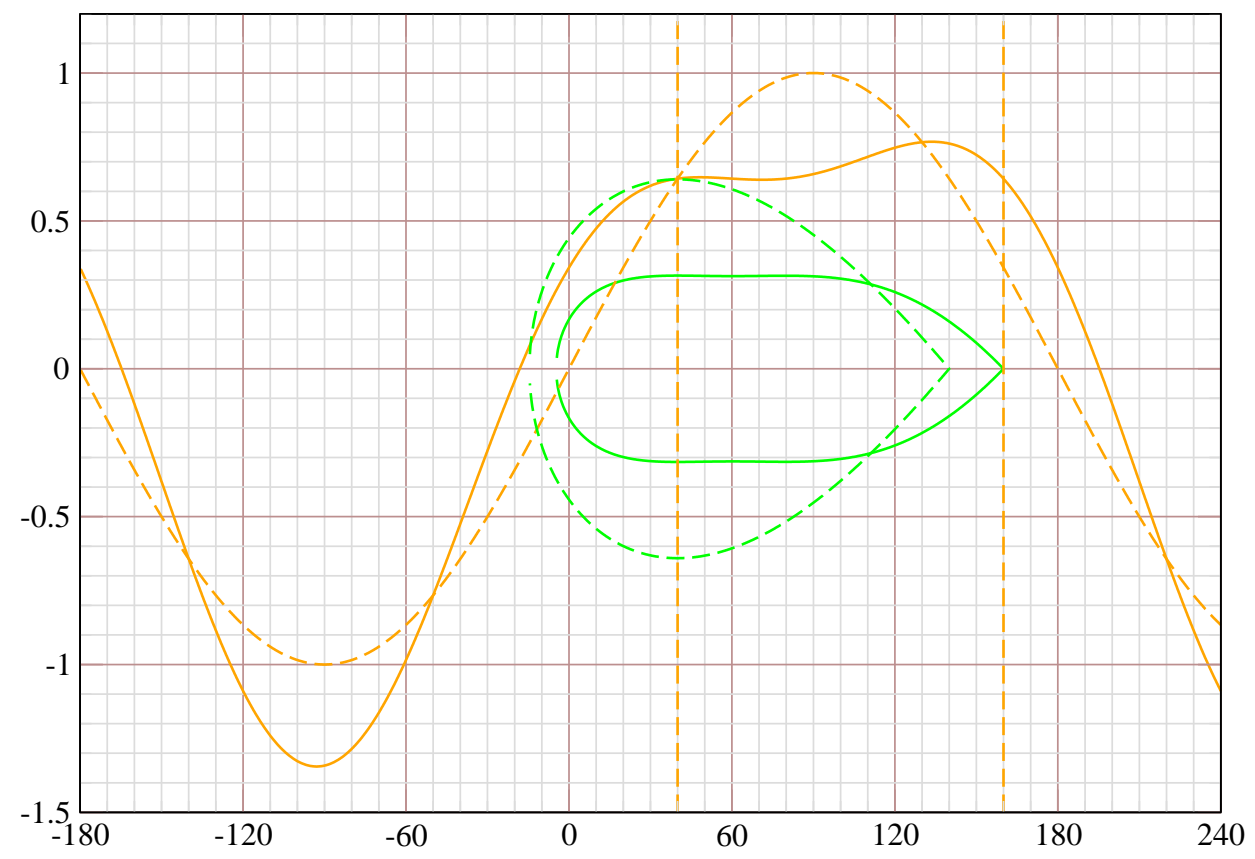

Figure 22: Same comparison but with a stable synchronous phase of 40 degrees. For the double harmonic setup, Flattened Bucket Option I has been used with $\phi_{s}=\phi_{1}=40^{\circ}, \psi_{0}=40^{\circ}$, and $V_{2} / V_{1}=0.347$. One can see by inspection that the double harmonic bucket area is significantly smaller than that of the single harmonic bucket. 


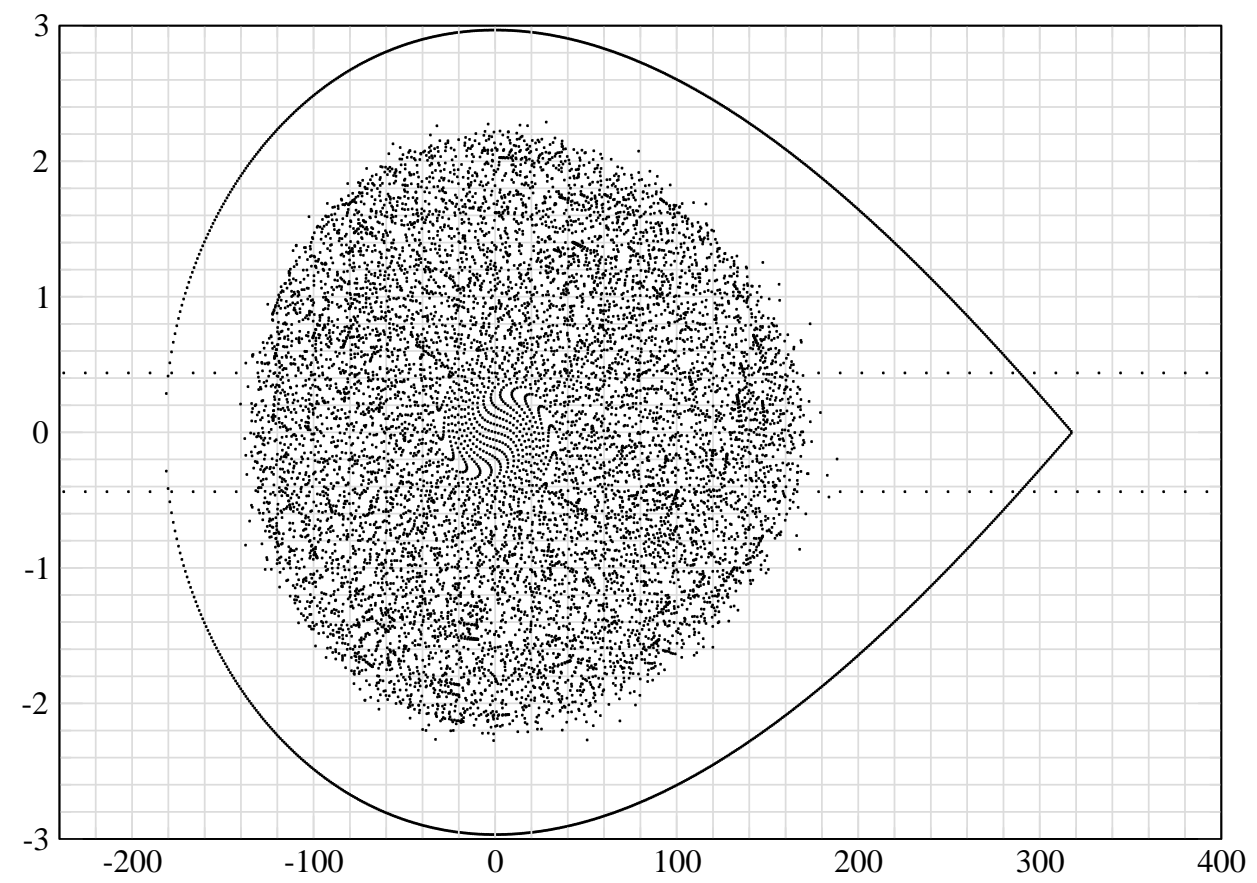

Figure 23: Result of simulated single harmonic acceleration of PP in Booster. Here a $1 \mathrm{eVs}$ uniform distribution of unbunched beam was captured as in Figure 12 and then accelerated to a synchronous proton kinetic energy of $386.8495 \mathrm{MeV}$. The corresponding revolution frequency is 1.04915084266 $\mathrm{MHz}$. The stable synchronous phase is 30 degrees. The units of the horizontal and vertical axes are nanoseconds and $\mathrm{MeV}$ respectively. The synchronous revolution period is 953.1518 nanoseconds. 


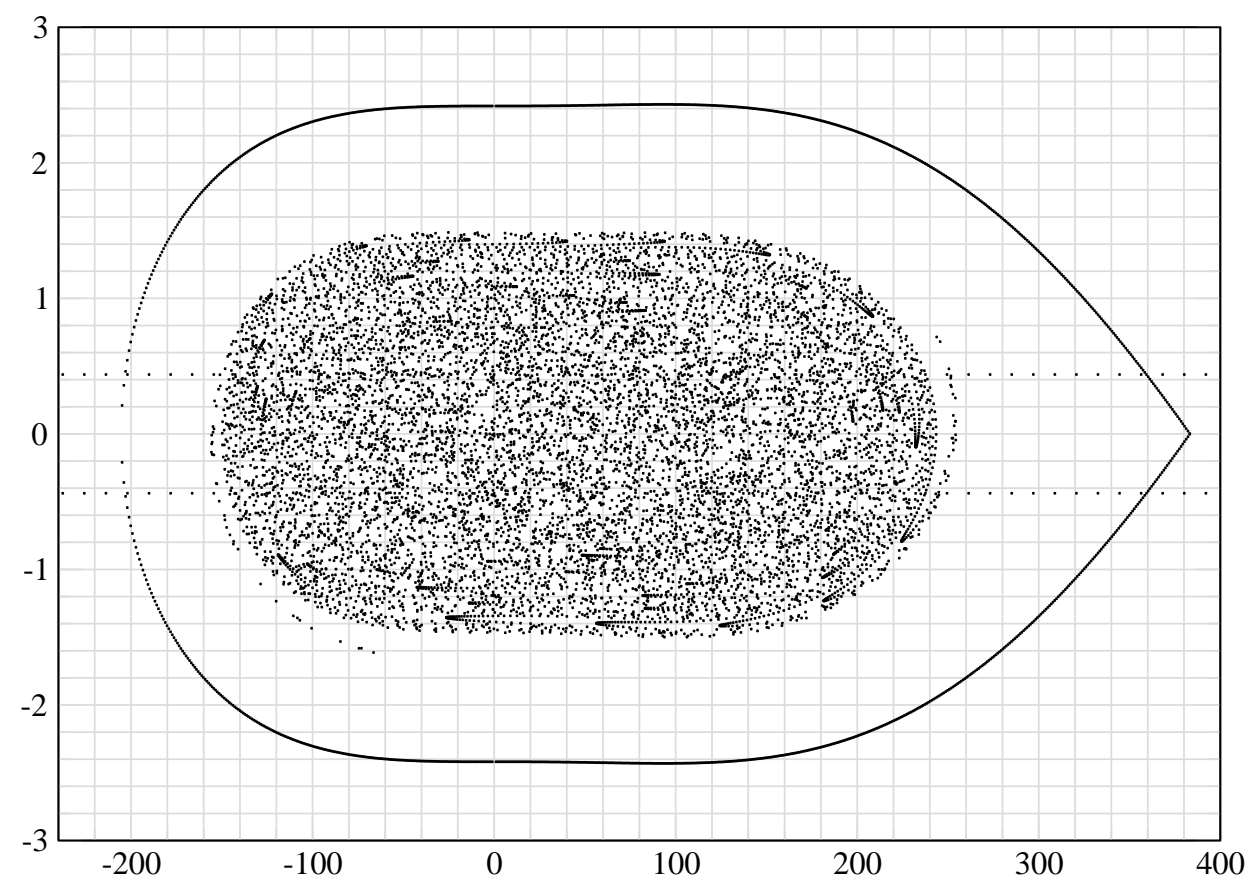

Figure 24: Result of simulated double harmonic acceleration of PP in Booster using Flattened Bucket Option I. Here a $1 \mathrm{eV} \mathrm{s}$ uniform distribution of unbunched beam was captured as in Figure 13 and again accelerated to a synchronous proton kinetic energy of $386.8495 \mathrm{MeV}$. The stable synchronous phase is again 30 degrees. Note that the bucket and bunch are not centered on the synchronous phase (which has time deviation zero in the figure), but the bunch is centered in the bucket. This is in accordance with Figure 5 and the findings of sections 5 and 6 . The units of the horizontal and vertical axes are nanoseconds and $\mathrm{MeV}$ respectively. The synchronous revolution period is 953.1518 nanoseconds. 


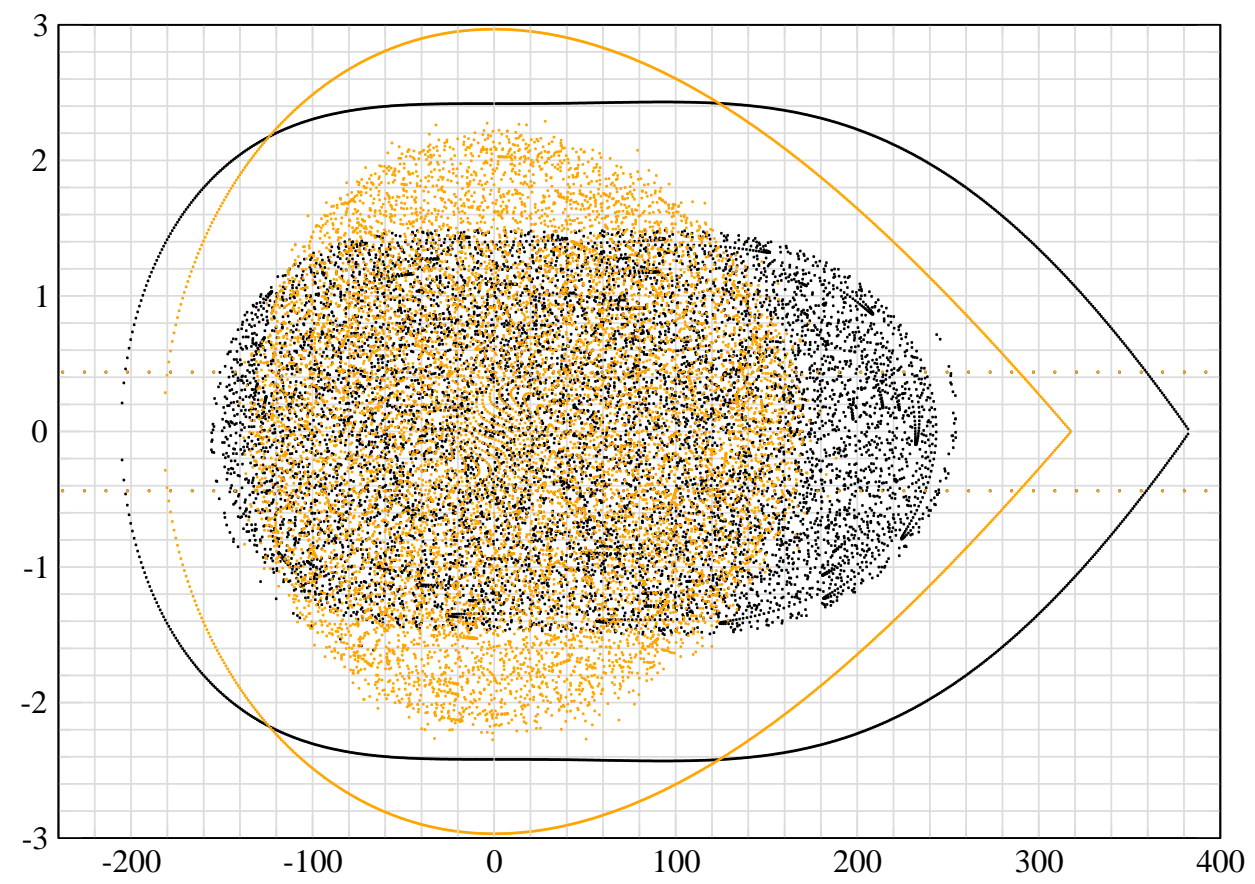

Figure 25: Here the simulation results of Figures $\mathbf{2 3}$ and $\mathbf{2 4}$ are shown together for comparison. The units of the horizontal and vertical axes are nanoseconds and $\mathrm{MeV}$ respectively. The synchronous revolution period is 953.1518 nanoseconds. 


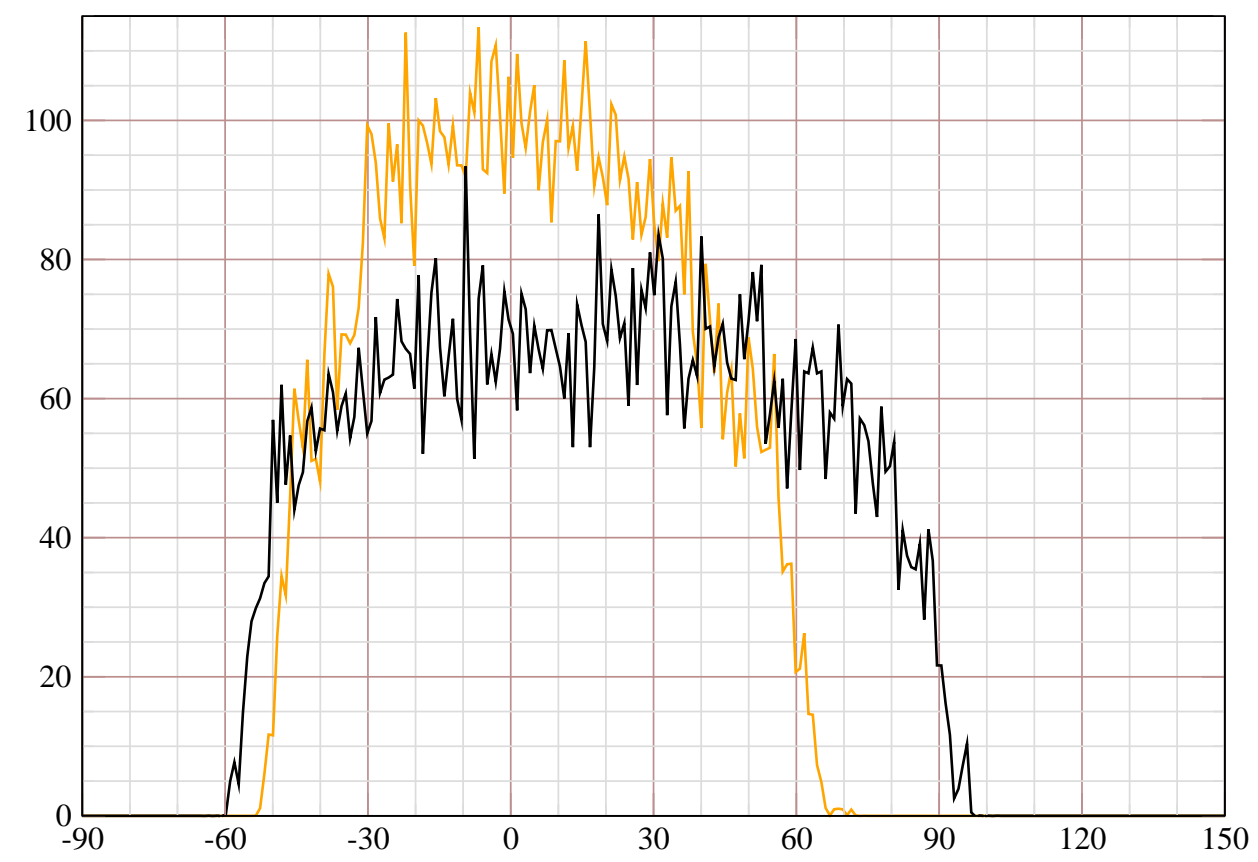

Figure 26: Binned data of Figure 25 showing that the double harmonic setup reduces the peak current by about 30\%. Here the horizontal axis gives the phase $\psi$ in degrees. 


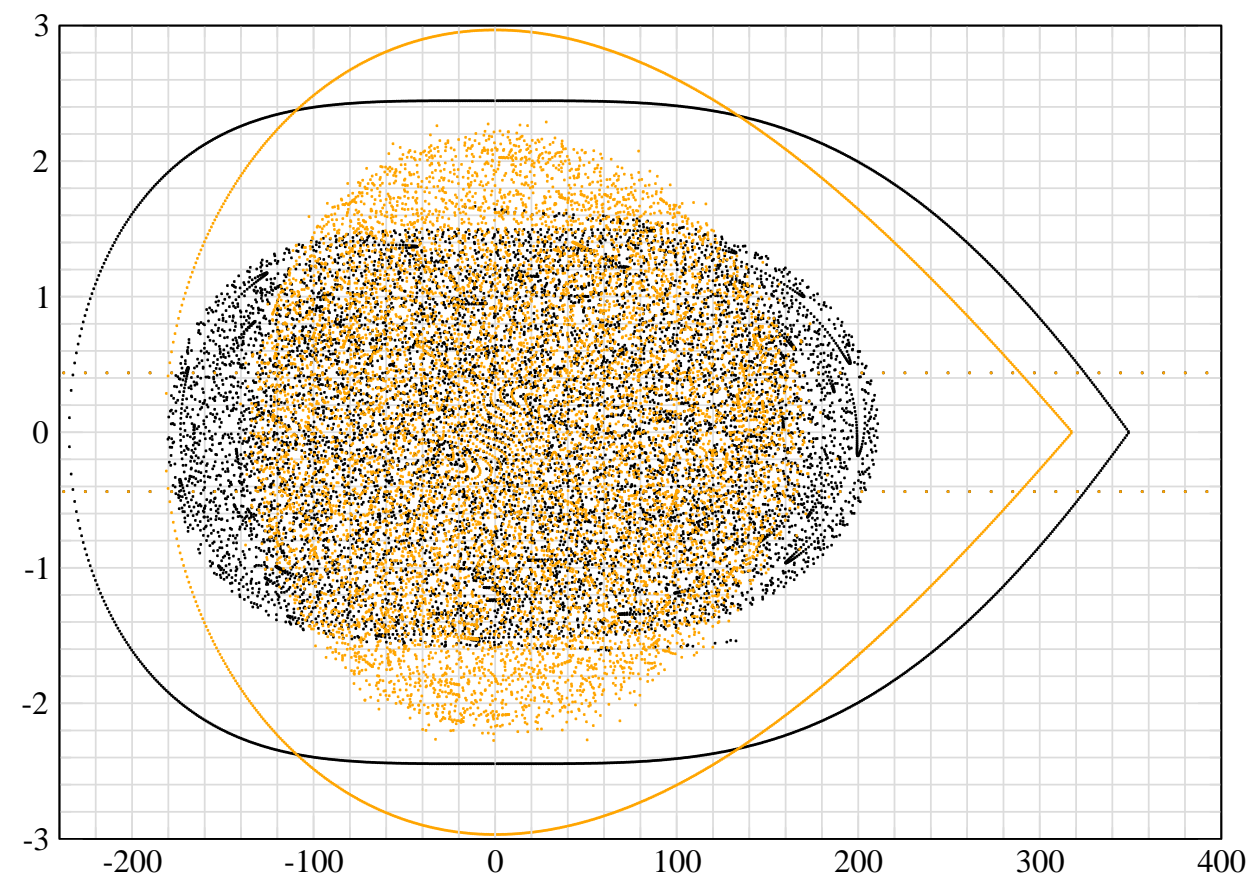

Figure 27: Comparison of the results of simulated single and double harmonic acceleration, but now with Flattened Bucket Option II used for the double harmonic setup. The synchronous proton kinetic energy is again $386.8495 \mathrm{MeV}$. The stable synchonous phase for the double harmonic setup is 41.8103 degrees. Note that the flattened bucket and bunch are now centered on the synchronous phase (which has time deviation zero in the figure). This is again in accordance with Figure $\mathbf{5}$ and the findings of sections 5 and 6 . The units of the horizontal and vertical axes are nanoseconds and $\mathrm{MeV}$ respectively. The synchronous revolution period is 953.1518 nanoseconds. 


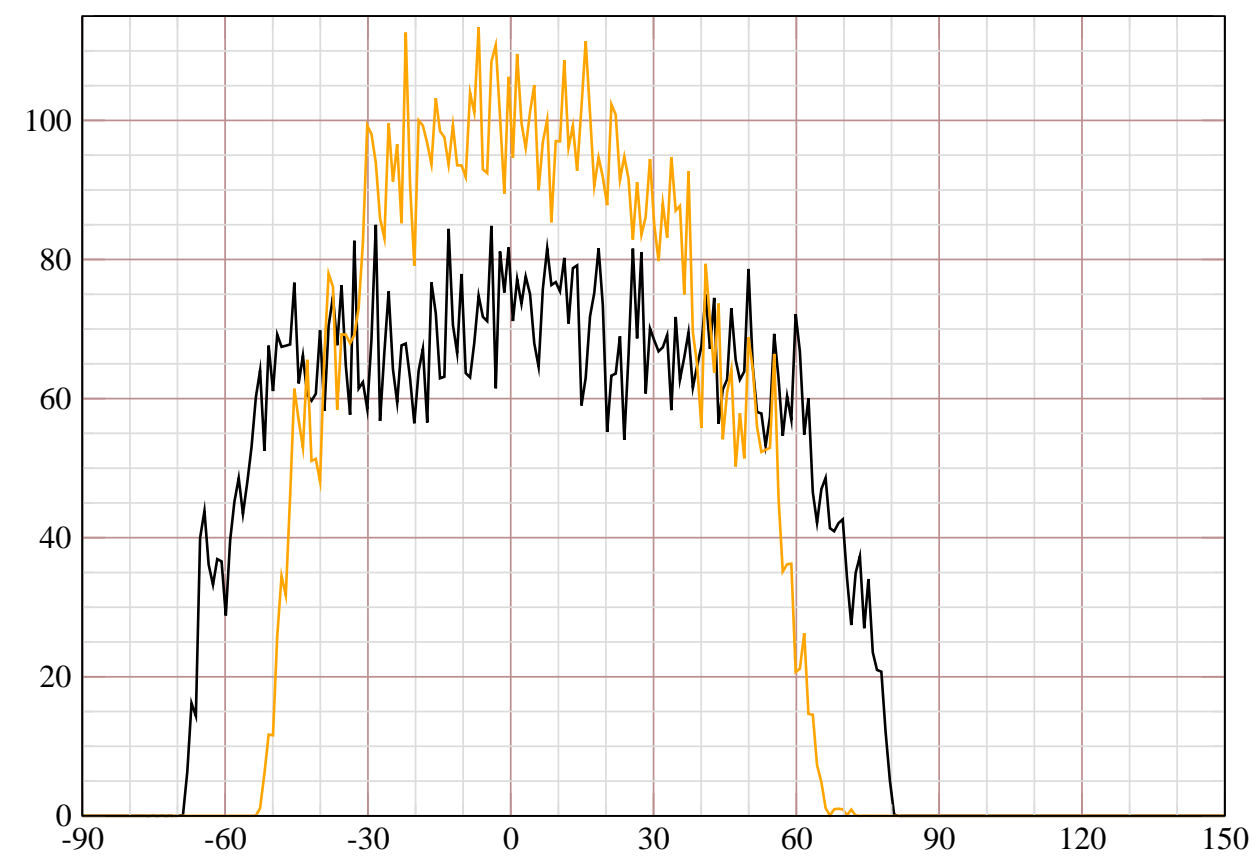

Figure 28: Binned data of Figure 27 showing again that the double harmonic setup reduces the peak current by about $30 \%$. The horizontal axis gives the phase $\psi$ in degrees. 


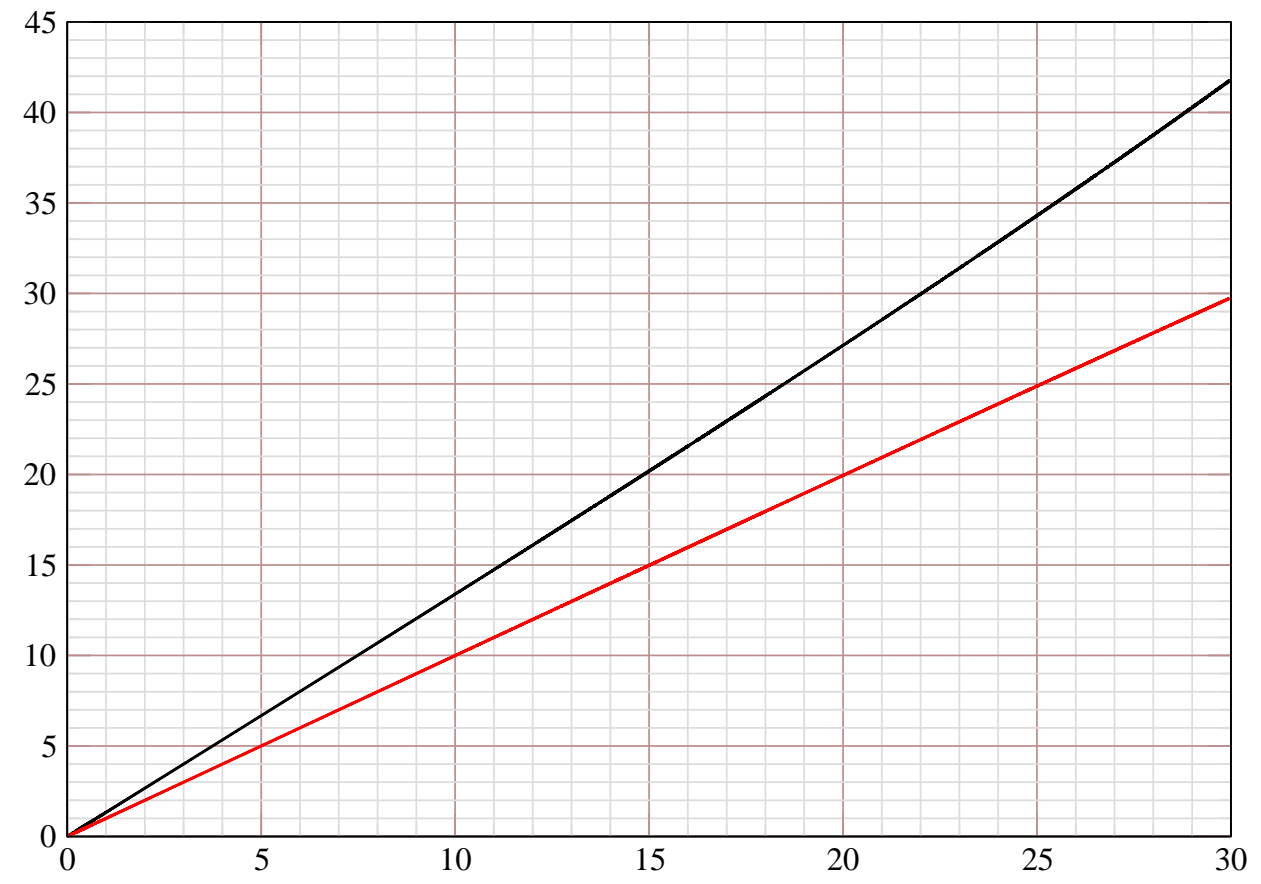

Figure 29: $\phi_{s}$ (black curve) and $\psi_{0}$ (red curve) obtained as functions of $\phi_{1}$ from (78) and (79) for Flattened Bucket Option II. Both axes are marked in degrees. The horizontal axis is $\phi_{1}$. 


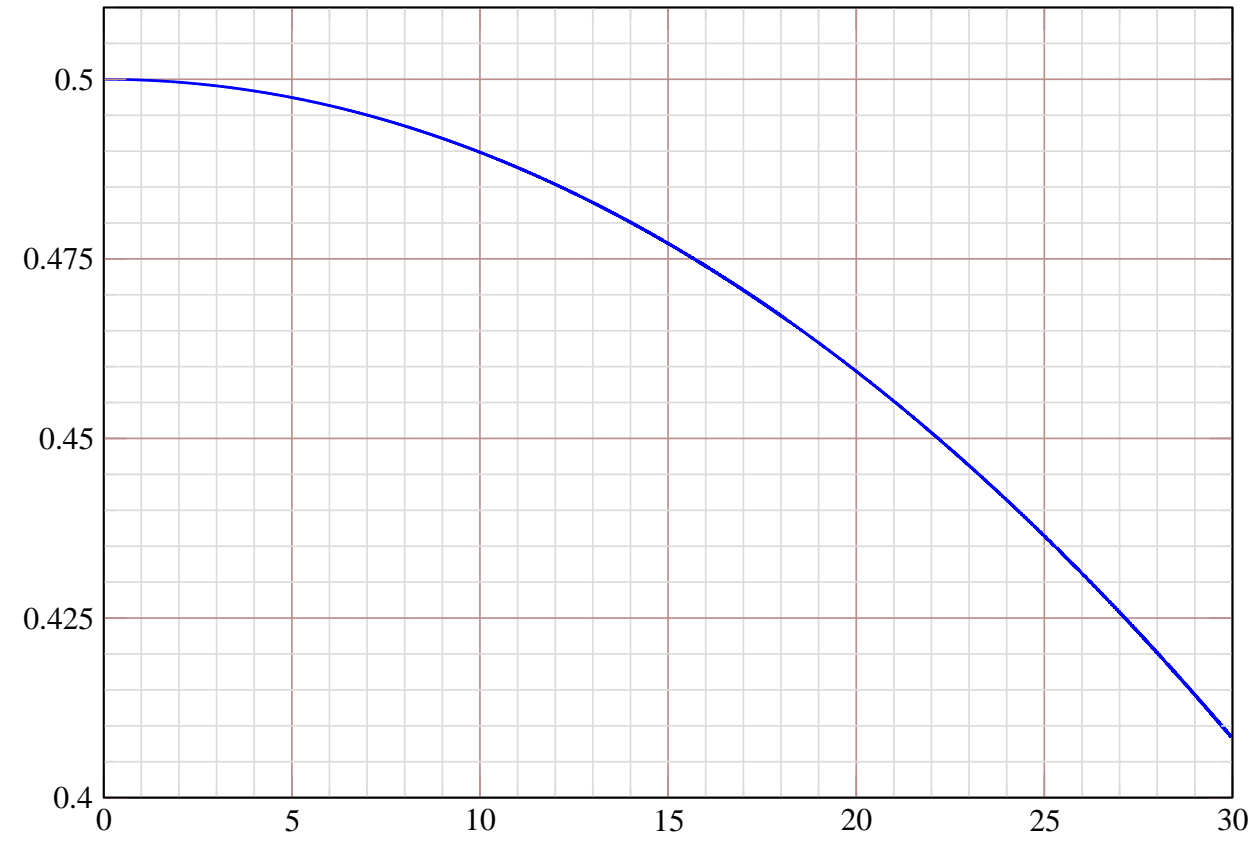

Figure 30: $V_{2} / V_{1}$ obtained from (78), (79), and (80) as a function of $\phi_{1}$ for Flattened Bucket Option II. The horizontal axis is $\phi_{1}$ marked in degrees. 


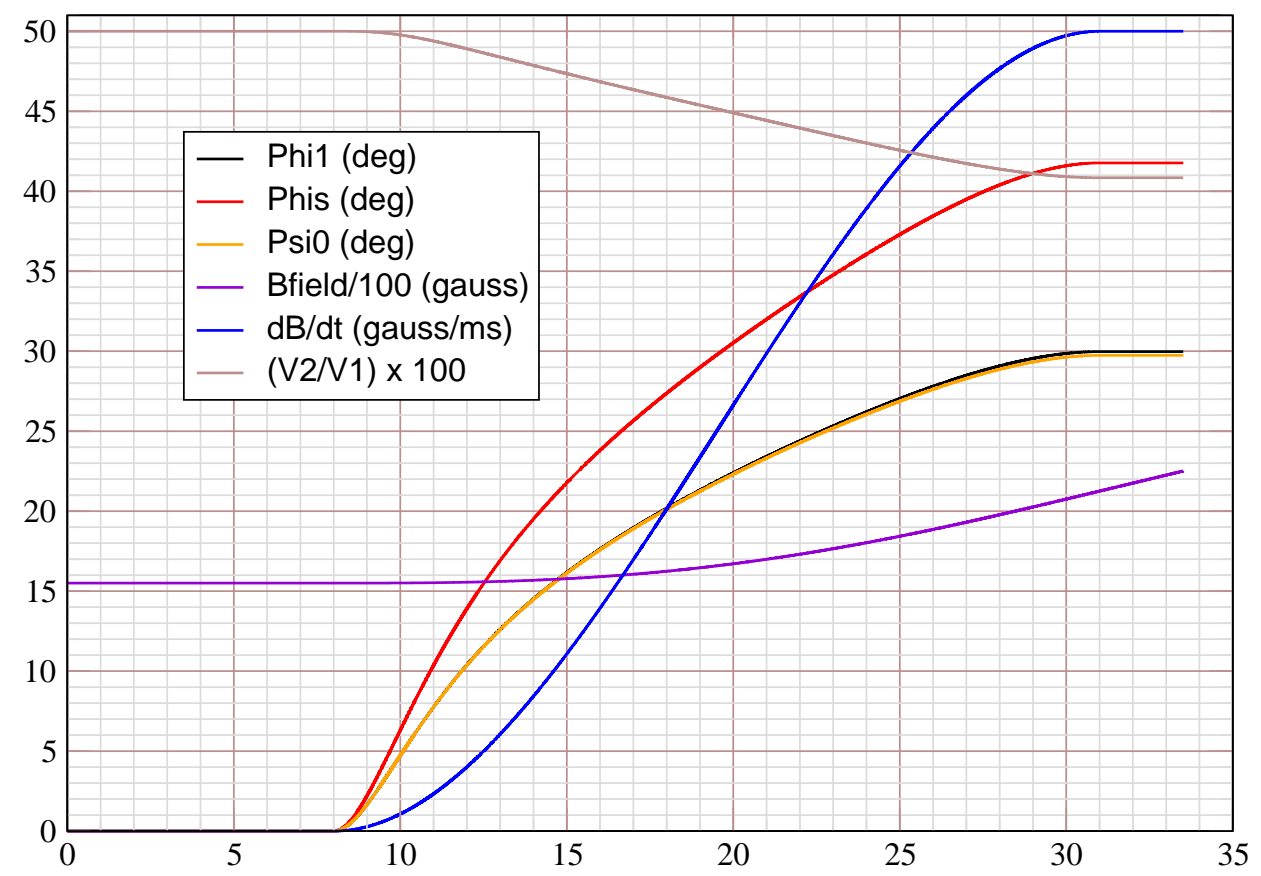

Figure 31: Phases $\phi_{1}, \phi_{s}, \psi_{0}$, and ratio $V_{2} / V_{1}$ along with the guide field $B$ and $d B / d t$ for capture of $\mathrm{PP}$ at $200 \mathrm{MeV}$ and acceleration to $386.8495 \mathrm{MeV}$ (kinetic energy) using Flattened Bucket Option II. The horizontal axis is marked in ms from injection. 